\title{
Circular RNA profiling reveals a potential role of hsa_circ_IPCEF1 in papillary thyroid carcinoma
}

\author{
MIN GUO $^{1 *}$, YUSHUANG SUN $^{2 *}$, JUNZHU DING $^{2}$, YONG LI $^{1}$, \\ SIHAN YANG ${ }^{1}$, YANNAZHAO ${ }^{1}$, XIN JIN $^{3}$ and SHAN-SHAN LI ${ }^{3}$ \\ ${ }^{1}$ Department of Endocrinology, The Fourth Affiliated Hospital of Harbin Medical University, \\ Harbin, Heilongjiang $150001 ;{ }^{2}$ College of Pharmacy, Harbin Medical University, Harbin, \\ Heilongjiang 150081; ${ }^{3}$ School of Medicine, Nankai University, Tianjin 300071, P.R. China
}

Received December 28, 2020; Accepted May 28, 2021

DOI: $10.3892 / \mathrm{mmr} .2021 .12241$

\begin{abstract}
Circular RNAs (circRNAs) are a novel type of non-coding RNAs that are expressed across species and are implicated in cellular biological processes, displaying dysregulated expression in various tumorigeneses. Therefore, circRNA deregulation could be a crucial event in thyroid carcinoma. The present study identified circRNA signatures in several patients with papillary thyroid carcinoma (PTC) to complement the understanding of PTC pathogenesis. Using microarray technology, the circRNA profiles in three pairs of PTC tumors and matching adjacent normal tissues were screened. Differentially expressed circRNAs were further validated by reverse transcription-quantitative PCR in whole blood from 57 pairs of subjects. Bioinformatics data analyses including miRNA response element prediction, Gene Ontology and Kyoto Encyclopedia of Genes and Genomes pathway, competing endogenous RNA and KEGG Orthology-Based Annotation System analyses were performed to predict circRNA associations with cancer-related putative downstream miRNAs and target genes. Receiver operating characteristic curves and the area under the curve (AUC) values were acquired to assess the performance of validated circRNAs in predicting potential associations with PTC. In total, 158 dysregulated circRNAs were identified in PTC tumors relative to adjacent normal tissues. Notably, one downregulated circRNA (hsa_circ_IPCEF1) showed the preferable predictive power $(\mathrm{AUC}=0.8010, \mathrm{P}<0.0001)$ and interactions with four cancer-related genes (CASR, CDC25B,
\end{abstract}

Correspondence to: Professor Xin Jin or Professor Shan-Shan Li, School of Medicine, Nankai University, 94 Weijin Road, Nankai, Tianjin 300071, P.R. China

E-mail: xin.jin@nankai.edu.cn

E-mail: shanshan.li@nankai.edu.cn

*Contributed equally

Key words: circular RNAs, papillary thyroid carcinoma, microarray assay, biomarkers
$\mathrm{NF \kappa B} 1$ and SHOC2). From these analyses, one PTC-related miRNA (hsa-miR-3619-5p) was identified as a potential target for hsa_circ_IPCEF1 sponging, indicating the hsa_circ_ IPCEF1/hsa-miR-3619-5p axis in pathogenesis.

\section{Introduction}

Thyroid cancer (TC) is the commonest endocrine malignancy worldwide, accounting for 1-5\% of all cancers in females and $<2 \%$ of all cancers in males. TC incidence continues to increase globally and is neither confined to a particular region nor influenced by underlying rates of TC (1). Papillary thyroid carcinoma (PTC) is the most frequent type of differentiated thyroid cancer, accounting for $80 \%$ of all pathological types of TC $(2,3)$. Studies on the incidence trends for TC subtypes and data from volumes 4-9 of Cancer Incidence Database on five continents (CI5) show that the incidence of PTC in both sexes has risen steadily in countries around the world over the past few decades (4-10). As indicated by a study of PTC in Manitoba, Canada, the age-standardized rate of PTC increased from $0.93 / 100,000$ to $6.68 / 100,000$ individuals per year over the period 1970-2010, showing a significant increase (11). At present, the main treatment for PTC is a combination of surgery, radioactive iodine and levothyroxine suppression, with a 50\% 5-year survival rate (12-15). Although PTC is less malignant than other cancer types, the incidence and recurrence rates are still high (20-40\%). The reasons for the apparent increase in PTC incidence are unclear and overdiagnosis and a small but actual increase in PTC incidence are indicated as the two main processes in some studies $(16,17)$. Considering the expanding demands for targeted therapies, the role of biomarkers in PTC diagnosis and treatment remains to be elucidated. BRAF mutation is proposed to be a prognostic biomarker; however, controversy exists regarding the high positive ratio of BRAF mutation combined with the low ratio of PTC aggressive biological behaviors (18-20). Recently, non-coding RNAs and their processing machinery have been shown to be common hallmarks of cancer regulating tumorigenesis, progression and metastasis via various mechanisms $(21,22)$. Micro (mi)RNA dysregulation has been discovered in numerous cancer types, including miRNA (miR)15a/16-1 cluster dysregulation in chronic lymphocytic 
leukemia, miR-127 in primary prostate cancer and bladder tumors and miR-29 family in lung cancer $(23,24)$. Significant advances have been made regarding the role of miRNAs in diagnostics, monitoring and therapy. For example, the expression levels of miR-145 are inversely correlated with BCR-ABL rearrangement levels during diagnosis and treatment of chronic myeloid leukemia, which can be used to modify the clinical treatment for improved outcomes (25). As potential oncogenes or oncosuppressor genes, miRNAs can be used as anticancer therapies. For instance, miR-203 inhibits the spheroid formation and cancer stem cells marker expression by targeting SOCS3, indicating a novel miRNA-based clinical treatment for estrogen receptor-positive breast cancer (26-28). In contrast to miRNAs, circular RNAs (circRNAs) are a special type of non-coding RNA molecules that are produced from pre-mRNA by backsplicing mechanisms. Unlike traditional linear counterparts (containing 5' and 3' ends), circRNAs have a closed circular structure, are not affected by RNA exonucleases in eukaryotes and exhibit distinct advantages in their stability over canonical linear RNAs $(29,30)$. Without free ends, circRNAs are expressed in a cell type-specific manner, display high stability and function in gene regulation in various pathologies. Recent studies have indicated that circRNAs can serve as miRNA sponges to regulate gene expression and sequester or recruit RNA/proteins; they even encode peptides or proteins $(31,32)$.

Increasing evidence shows that circRNAs are stably expressed in exosomes, plasma and saliva, indicating their high capacity for detection and clinical utility in cancer $(33,34)$. Evidence suggests that circRNA profiles are cancer specific and related to tumor occurrence and progression (35-37). The present study screened circRNA expression in PTC tumors and verified the dysregulated circRNAs in whole blood from patients with PTC to evaluate their potential value as PTC biomarkers. The possible functional associations of these circRNAs with PTC were also analyzed using bioinformatics.

\section{Materials and methods}

Ethics. The present study was approved by the Medical Ethics Committee of Harbin Medical University (approval no. IRB3011619). All participants involved in this study were informed of the research objectives and signed consent forms before the study.

Subjects. Patients who underwent thyroidectomy were recruited at The First, Second and Fourth Clinical Hospitals Affiliated with Harbin Medical University between January 2017 and July 2019. Patients with PTC were diagnosed and evaluated by neck ultrasonic imaging and pathological diagnosis. Histopathological analysis and diagnostic immunohistochemistry of all thyroid tissue specimens were independently performed by two licensed pathologists. The immunohistochemistry of NF- $\kappa \mathrm{B} 1$ was used to confirm the diagnostic accuracy. Briefly, the morphological and pathological identification of PTC was performed by H\&E staining at room temperature for $10 \mathrm{~min}$. Paraformaldehyde-fixed $\left(4^{\circ} \mathrm{C}\right.$ overnight) and paraffin embedded thyroid tissue slides were dewaxed in xylene and rehydrated in ethanol. The endogenous peroxidase was blocked by $3 \% \mathrm{H}_{2} \mathrm{O}_{2}$ at room temperature for $10 \mathrm{~min}$. Heated slides in a microwave submersed in $1 \mathrm{X}$ citrate unmasking solution (Cell Signaling Technology, Inc.) until boiling was initiated, followed by $10 \mathrm{~min}$ at sub-boiling temperature $\left(95-98^{\circ} \mathrm{C}\right)$. The slides were cooled on bench top for $30 \mathrm{~min}$. The slides were then incubated with a 1:100 dilution of $\mathrm{NF}-\kappa \mathrm{B} 1$ primary antibody (cat. no. bs-1194R; Beijing Biosynthesis biotechnology Co., Ltd.) at $4^{\circ} \mathrm{C}$ overnight. After washing, the slides were incubated with goat anti-rabbit IgG (cat. no. PV-6001; OriGene Technologies, Inc.) for $20 \mathrm{~min}$, followed by staining with DAB for $5 \mathrm{~min}$ at room temperature. The slides were counterstained with hematoxylin at room temperature for $20 \mathrm{sec}$, and then dehydrated with ethanol and xylene. The morphological changes were observed by a light microscope (magnification, x40 or x100; BM2000; Nanjing Jiangnan Yongxin Optics Co., Ltd.). Images were captured in three random fields. The number of cells was analyzed by ImageJ software (v1.8.0.112, National Institutes of Health). Ultimately, 57 cases of PTC and age- and sex-matched healthy controls were recruited for this study (Table I). Among these subjects, three pairs of PTC tumors and matching paracancerous tissues were collected for microarray analysis. In total, 57 pairs of whole-blood samples were obtained from all subjects and refrigerated at $-80^{\circ} \mathrm{C}$ for further studies.

RNA isolation and quality control. Total RNA was extracted from the tissue samples and whole blood with TRI Reagent BD, which was supplied by Sigma-Aldrich (Merck KGaA) in accordance with the manufacturer's protocol. RNA was precipitated with isopropanol, washed twice with $75 \%$ ethanol and resuspended in RNase/DNase-free water. The RNA concentration and purity were assessed with a Nano-200 system (Hangzhou Allsheng Instruments Co., Ltd.). RNA samples with OD260/OD280 ratios $\geq 1.8$ and $\leq 2.1$ and OD260/OD230 ratios $>1.8$ were used for further studies.

circRNA array. circRNA array analysis was performed by KangChen Bio-tech. Briefly, three pairs of PTC tumors and matching paracancerous tissues were used for Arraystar Human circRNA Array analysis. The linear RNAs were removed by RNase R (Epicenter Biotechnologies) to enrich circRNAs. The enriched circRNAs were amplified and transcribed into fluorescent complementary RNA (cRNA) by the random priming method [Quick Amp Labeling Kit, One-Color (Agilent p/n 5190-0442)], which was performed by KangChen Bio-tec. Subsequently, these labeled cRNAs were purified by an RNeasy Mini kit (Qiagen $\mathrm{GmbH}$ ) and were hybridized to an Arraystar human circular RNA array V2 (8x15K, Arraystar, Inc.). The arrays were then scanned with an Agilent G2505C scanner (Agilent Technologies, Inc.) followed by washing. Array images and data analysis were performed using Agilent Feature Extraction software (version 11.0.1.1; Agilent Technologies, Inc.) and the $\mathrm{R}$ software package version 3.1.2 ( $\mathrm{R}$ Foundation for Statistical Computing; http://www.R-project.org/) Differentially expressed circRNAs with P-values $<0.05$ between the two groups were visualized in a volcano plot and with hierarchical clustering. The relevant datasets have been submitted to National Center for Biotechnology Information Gene Expression Omnibus (GEO). 
Table I. Correlation between hsa_circ_IPCEF1 expression and clinicopathological characteristics in 57 patients with papillary thyroid carcinoma.

\begin{tabular}{|c|c|c|c|}
\hline Characteristics & Number of patients (\%) & Relative expression & P-value \\
\hline \multicolumn{4}{|l|}{ Age (years) } \\
\hline$<45$ & $26(45.6)$ & $0.75 \pm 0.16$ & \multirow[t]{2}{*}{0.1933} \\
\hline$\geq 45$ & $31(54.4)$ & $0.52 \pm 0.09$ & \\
\hline \multicolumn{4}{|l|}{ Sex } \\
\hline Male & $17(29.8)$ & $0.76 \pm 0.20$ & \multirow[t]{2}{*}{0.1571} \\
\hline Female & $40(70.2)$ & $0.45 \pm 0.11$ & \\
\hline \multicolumn{4}{|l|}{ Tumor size $(\mathrm{cm})$} \\
\hline$<2$ & $49(86.0)$ & $0.70 \pm 0.14$ & \multirow[t]{2}{*}{0.1246} \\
\hline$\geq 2$ & $8(14.0)$ & $0.17 \pm 0.08$ & \\
\hline \multicolumn{4}{|l|}{ TNM stage } \\
\hline I-II & $42(73.7)$ & $0.77 \pm 0.20$ & \multirow[t]{2}{*}{0.1515} \\
\hline III-IV & $15(26.3)$ & $0.28 \pm 0.11$ & \\
\hline \multicolumn{4}{|l|}{ Multifocality } \\
\hline Yes & $30(52.6)$ & $0.61 \pm 0.09$ & \multirow[t]{2}{*}{0.4660} \\
\hline No & $27(47.4)$ & $0.48 \pm 0.16$ & \\
\hline \multicolumn{4}{|c|}{ Extrathyroidal extension } \\
\hline Yes & $12(21.1)$ & $0.69 \pm 0.26$ & \multirow[t]{2}{*}{0.8503} \\
\hline No & $45(78.9)$ & $0.63 \pm 0.18$ & \\
\hline \multicolumn{4}{|l|}{ LNM } \\
\hline Yes & $32(56.1)$ & $0.82 \pm 0.13$ & \multirow[t]{2}{*}{$<0.001^{\mathrm{a}}$} \\
\hline No & $25(43.9)$ & $0.25 \pm 0.05$ & \\
\hline
\end{tabular}

${ }^{\mathrm{a}} \mathrm{P}<0.001$. circ, circular RNA; TNM, tumor-node-metastasis; LNM, lymph node metastasis.

The GEO accession no. is GSE173299 (https://www.ncbi.nlm. nih.gov/geo/query/acc.cgi?acc=GSE173299).

Reverse transcription-quantitative (RT-q) PCR validation. The top 20 most differentially expressed circRNAs from array analysis were further validated by RT-qPCR on 57 pairs of whole blood and three pairs of tissue samples from subjects. Total RNA $(1 \mu \mathrm{g})$ was reverse-transcribed into cDNA using a First Strand cDNA Synthesis kit (Thermo Fisher Scientific, Inc.) in accordance with the manufacturer's protocol. The candidate circRNAs were amplified by Power SYBR Green PCR Master Mix (Applied Biosystems; Thermo Fisher Scientific, Inc.) with various primers. The primers for each circRNA are presented in Table II. RT-qPCR was performed on an ABI 7300 Real-Time PCR System (Applied Biosystems; Thermo Fisher Scientific, Inc.) in a $10 \mu \mathrm{l}$ PCR mixture. The thermocycling conditions were as follows: $95^{\circ} \mathrm{C}$ for $10 \mathrm{~min}$, 40 cycles of $95^{\circ} \mathrm{C}$ for $15 \mathrm{sec}$ and $60^{\circ} \mathrm{C}$ for $60 \mathrm{sec}$. $\beta$-actin was used as an internal control. The relative expression of each group was analyzed using the $2^{-\Delta \Delta \mathrm{Cq}}$ method (38).

Circularization of hsa_circ_IPCEFl validation. To confirm the splicing junction of hsa_circ_IPCEF1, RT-PCR followed by sequencing was performed in whole blood from PTC subjects. Briefly, total RNA was extracted from whole blood from patients with PTC as described above. After reverse transcription, the cDNA was amplified using PrimeSTAR ${ }^{\circledR}$ Max DNA Polymerase (Takara Biotechnology Co., Ltd.). The thermocycling conditions were 35 cycles of $95^{\circ} \mathrm{C}$ for $10 \mathrm{sec}$, $55^{\circ} \mathrm{C}$ for $15 \mathrm{sec}$ and $72^{\circ} \mathrm{C}$ for $10 \mathrm{sec}$. Using divergent primers (forward: 5'-GTTTGTCTGCTGCTGAAGATGAG-3'; reverse 5'-CCATCAGCTTTCTCTGCCTTTGTC-3') to amplify the hsa_circ_IPCEF1 product containing a head-to-tail splicing junction site. The PCR product was purified by $1.5 \%$ agarose gel electrophoresis, visualized by ethidium bromide and identified by Sanger sequencing.

Competing endogenous RNA (ceRNA) network analysis. An interaction analysis of circRNA/miRNA was performed by KangChen Bio-tech based on TargetScan (39-41). Through merging the commonly targeted miRNAs, a circRNA-miRNA-mRNA interaction network of hsa_circ_ IPCEF1 was constructed by Cytoscape 3.7.2 (https://cytoscape. org). Briefly, by merging the commonly targeted miRNAs, a ceRNA network was constructed via three conditions (42). First, the relative concentration of the ceRNAs and their microRNAs is clearly important; second, the effectiveness of a ceRNA depends on the number of microRNAs that it can 'sponge'; and third, not all of the MREs on ceRNAs are equal. Therefore, only these ceRNA-pair relations that passed some filtering measures were accepted. In addition to a measure with the number of common microRNAs, a hypergeometric 
Table II. Primers fortop 20 dysregulated circRNAs.

\begin{tabular}{|c|c|c|c|}
\hline circRNA & Regulation & Forward & Reverse \\
\hline hsa_circ_0000277 & Up & 5'CAGTCTTCAAGGTGGGATCGTAA3' & 5'TGGAAGGCTTGGATCAGTCAG3' \\
\hline hsa_circ_0044556 & Up & 5'CTGGTCCTGATGGCAAAACTG3' & 5'GGGGTCCTTGAACACCAACA3' \\
\hline hsa_circ_0014234 & Up & 5'CCAGAGCTATGCTTTAGGTCTCA3' & 5'AGTGGGAAGTGGGAGGTGTC3' \\
\hline hsa_circ_0040773 & Up & 5'AAGTATTACCCCGTCTTTAAGCAG3' & 5'TTCCAGACACGCCCATCAC3' \\
\hline hsa_circ_0074530 & Up & 5'ATGAGCAGGCACTCCTTGGA3' & 5'TCAGTGGCGGGTACACCTTC3' \\
\hline hsa_circ_0074595 & Up & 5'GCCTATAAGGAGGACTATCACAAG3' & 5'CTGCGGTGCGTGATGATA3' \\
\hline hsa_circ_0001681 & Up & 5'AGAGGTGGCATCTGTGAACTGTC3' & 5'GGGAAGGCGTATGTTCAAGGTA3' \\
\hline hsa_circ_0049237 & Up & 5'CCATAGGCTCACAACACCACA 3' & 5'CCCTGCGTGTCCACCTCTA3' \\
\hline hsa_circ_0058230 & Up & 5'TGGATGGGGAGCCCTACAAG3' & 5'CCAGGTGCGGGTGTACAGG3' \\
\hline hsa_circ_0057691 & Up & 5'AGCAACCAAGTGCCAGGAGT3' & 5'CGGGTGCATCTGTCACATAACT3' \\
\hline hsa_circ_FGFR2 & Down & 5'GAATACGGGTCCATCAATCACA3' & 5'ATCACGGCGGCATCTTTC3' \\
\hline hsa_circ_0095448 & Down & 5'CCCCTGGAATAACATACAAACC3' & 5'GTAGCTGCTCCCGTAAACTGAT3' \\
\hline hsa_circ_0031968 & Down & 5'GTTTGGTGTCTCCCCGCTAT3' & 5'GCCTTCTGCAACTGGAATCA3' \\
\hline hsa_circ_IPCEF1 & Down & 5'AGATAAGCCTGCTGGATCAAAG3' & 5'ACAGTGAAATCAGGCAGGTTG3' \\
\hline hsa_circ_0021549 & Down & 5'TTCGGAGGTAACAGTGAAGGGA3' & 5'AGGCATCTGGATACCATCTGTTCT3' \\
\hline hsa_circ_0070098 & Down & 5'GCTCAGTGTCAGCCTCTATTTTG3' & 5'GCATTGGTTGGCAGCTATTTG3' \\
\hline hsa_circ_0079891 & Down & 5'GAATTGCTTTTGATGCTGAGTCTG3' & 5'TTTCCATGAGTTTGGGGTAGG3' \\
\hline hsa_circ_0001938 & Down & 5'CATCGTTCTTACAGTTCTGCACA3' & 5'CAGCCACGAAGCCAAAGC3' \\
\hline hsa_circ_0020396 & Down & 5'GCTTGATCGAAATCGTCCACA3' & 5'GAAAGTTCATCCGCTCCTCTG3' \\
\hline hsa_circ_0021550 & Down & 5'GATTCGGAGGTAACAGTGAAGG3' & 5'TACAGAGCAAAAGATGGAAGCA3' \\
\hline$\beta$-actin & & 5'GTGGCCGAGGACTTTGATTG3' & 5'CCTGTAACAACGCATCTCATATT3' \\
\hline
\end{tabular}

circRNA/circ, circular RNA.

test was executed for each ceRNA pair separately, which was defined by four parameters: i) $\mathrm{N}$ is the total number of miRNAs used to predict targets; ii) $\mathrm{K}$ is the number of miRNAs that interact with the chosen gene of interest; iii) $n$ is the number of miRNAs that interact with the candidate ceRNA of the chosen gene; and iv) is the common miRNA number between the two genes (43). The test calculates the P-value by using the following formula:

$$
P=\sum_{i=c}^{\min (K, n)} \frac{\left(\begin{array}{c}
K \\
i
\end{array}\right)\left(\begin{array}{c}
N-K \\
n-i
\end{array}\right)}{\left(\begin{array}{c}
N \\
n
\end{array}\right)}
$$

Functional group analysis. The molecular functional roles of the circRNA-target gene profiles were analyzed using Gene Ontology (http://www.geneontology.org), including biological process (BP), cellular component (CC) and molecular function (MF). The P-value produced by topGO (http://www.bioconductor.org/packages/release/bioc/html/topGO.html) denoted the significance of GO term enrichment in the circRNA-miRNA targeted genes. Kyoto Encyclopedia of Genes and Genomes (KEGG) pathway analysis was then performed (https://www. genome.jp/kegg/). The P-value (EASE score, Fisher P-value, or hypergeometric P-value) denoted the significance of the pathway relevant to PTC. The P-value cutoff was 0.05 . The ceRNAs of each differentially expressed circRNA were further annotated in terms of the diseases and pathways using the KEGG orthology-based annotation system (KOBAS), (version 3.0; http://kobas.cbi.pku.edu.cn/kobas3/?t=1). $\mathrm{P}<0.05$ was considered to indicate a statistically significant difference.
Statistical analysis. All data are expressed as the means \pm standard error of the mean. circRNAs with fold changes $\geq 2$ and P-values $\leq 0.05$ were selected as the significantly differentially expressed circRNAs. Receiver operating characteristic (ROC) curves and the area under the curve (AUC) value and $95 \%$ confidence intervals (CIs) were established using GraphPad Prism 8.0 (GraphPad Software, Inc.) to predict their potential association with PTC. Comparisons of data were acquired by one-way ANOVA followed by Bonferroni post hoc or Student's t-test. $\mathrm{P}<0.05$ was considered to indicate a statistically significant difference.

\section{Results}

Characterization of human thyroid samples. A total of three pairs of PTC tumors and matching paracancerous tissues were collected for microarray. The PTC tumor showed the typical papillary architecture with branching and the enlarged irregular nuclei were oval-shaped and overlapping, showing ground-glass appearance with prominent nuclear grooves and pink cytoplasmic invaginations and intra-nuclear pseudoinclusion (Fig. 1A). NF- $\kappa \mathrm{B} 1$-related cancer phenotype (increased expression of $\mathrm{NF}-\kappa \mathrm{B} 1$ in cell cytoplasm) were also observed in PTC tumors (Fig. 1B and C). Through pathological reports, the tumor tissues were confirmed to be PTC for circRNA chip analysis.

circRNA expression profile in PTC. circRNA profiling was performed with Arraystar Human circRNA Array in three pairs 
A
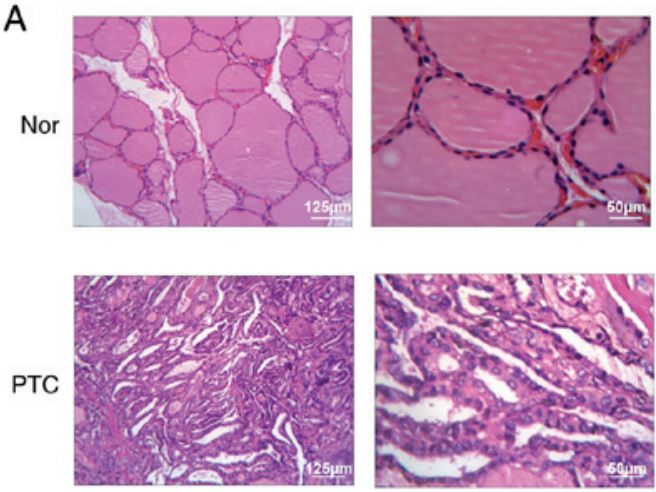

B
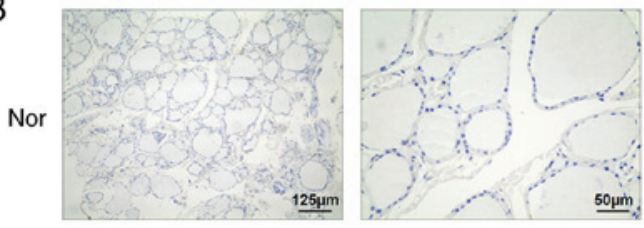
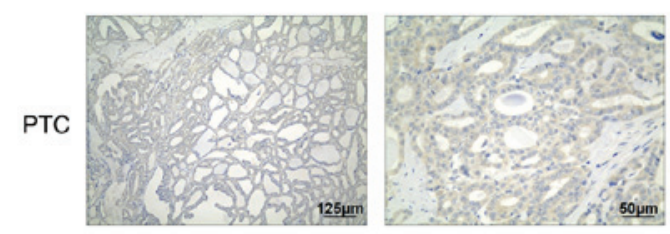

C

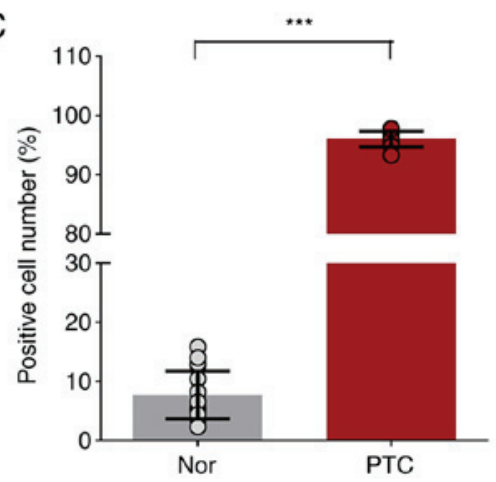

Figure 1. Immunohistochemistry of thyroid specimens. Hematoxylin-eosin stained images of (A) papillary thyroid cancer and cancer contralateral normal thyroid tissues, (B) NF-kB1 staining in papillary thyroid cancer and cancer contralateral normal thyroid tissues, (C) NF- $\mathrm{B} 1$ expression in papillary thyroid cancer and cancer contralateral normal thyroid tissues. Results are described as ratios of NF-kB1 positive cells to all cells. ${ }^{* * *} \mathrm{P}<0.001$ ( $\mathrm{n}=3$ pairs of samples; $\leq 6-10$ fields from each group were imaged and scored in a blinded manner). Comparisons of data were acquired by one-way ANOVA followed by Bonferroni post hoc. Magnification of x40 and x100 from left to right. Nor, PTC tumor-adjacent tissues; PTC, papillary thyroid cancer tissues.

of PTC tumor tissues and cancer contralateral normal thyroid tissues. Hierarchical cluster analysis revealed that the circRNA expression patterns were distinctive between PTC tumor and tumor-adjacent normal thyroid tissues (Fig. 2A). A cut-off value for differential expression fold change was set to 2.0 and 158 significantly dysregulated circRNAs (74 upregulated and 84 downregulated) were found in the PTC tumors (Fig. 2B-D, $\mathrm{P}<0.05$ and false discovery rate $<0.05$ ).

Validation of the differential expression of circRNAs in $P T C$. From microarray results, thetop 20 most dysregulated circRNAs (10 upregulated and 10 downregulated, Table II) were selected for RT-qPCR validation on three pairs of PTC tumor tissues and cancer contralateral normal thyroid tissues as well as in whole blood from 57 pair of patients with PTC and healthy controls. The characteristics of the 57 patients with PTC are presented in Table I. The RT-qPCR results showed that 7 circRNA expression trends were consistent with the microarray assay, which included 3 upregulated circRNAs (hsa_circ_0000277,hsa_circ_0074530 and hsa_circ_0057691) and 4 downregulated circRNAs (hsa_circ_0020396, hsa_circ_0095448,hsa_circ_IPCEF1 and hsa_circ_0021549; Figs. 3 and 4).

Functional annotation of the dysregulated circRNAs. By targeting miRNA response elements (MREs), circRNAs can regulate mRNA by acting as miRNA sponges. The predicted MREs for these seven validated circRNAs are presented in Table III. Based on TargetScan, the targets/miRNAs were analyzed to identify the potential targets of miRNAs (KangChen Bio-Tech). Based on the seven dysregulated circRNAs and their predicted MREs, the 256 predicted ceRNA genes are listed in Table SI.

The molecular functional roles of the circRNA-target gene profiles were analyzed using GO and KEGG analyses. For the seven dysregulated circRNAs, the top 10 enriched GO terms are shown and ranked by Enrichment Score [-log10 (P-value)]. Through GO analysis of the different genes of dysregulated circRNAs, it is possible to identify genes that may be related to changes in gene function in PTC pathogenesis. As a result, the enriched MF, BP and CC terms were determined to be involved in 'Olfactory receptor activity', 'G-protein coupled receptor signaling pathway' and 'Signal receptor activity in the plasma membrane' (Fig. 5A). In KEGG analysis, the significantly enriched pathways were involved in the "cytosolic DNA-sensing pathway', the 'RIG-I-like receptor signaling pathway' and the 'Olfactory transduction' pathway (Fig. 5B). These results indicate that these dysregulated circRNAs may cause PTC cells to undergo a series of molecular and signal changes.

ceRNA analyses of dysregulated circRNAs. Among these potential circular RNAs, hsa_circ_IPCEF1 was related to 207 ceRNA genes out of the 256 ceRNA genes, exhibiting the strongest role in gene regulation (Table SII; Fig. 6A). Therefore, 207 ceRNA genes related to hsa_circ_IPCEF1 
A

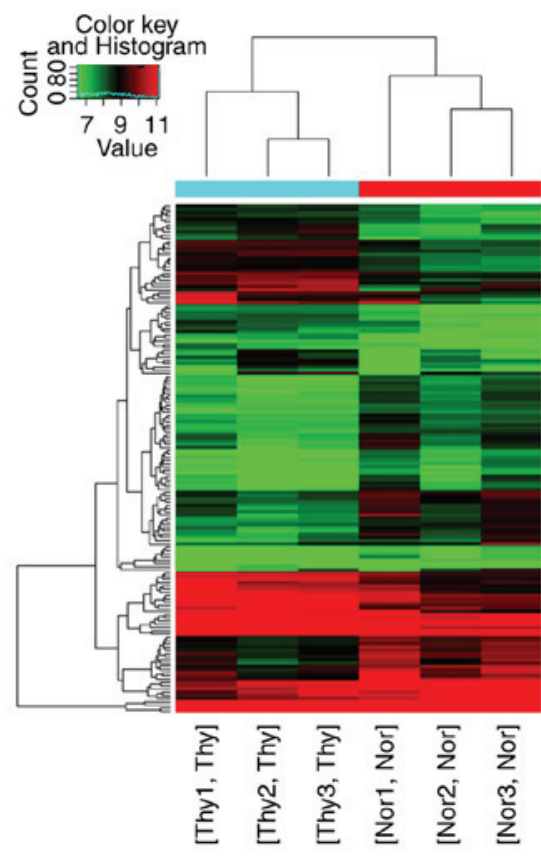

C

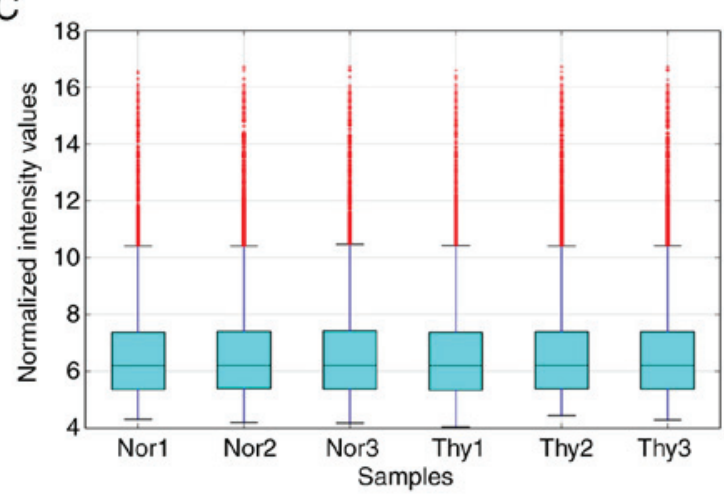

B

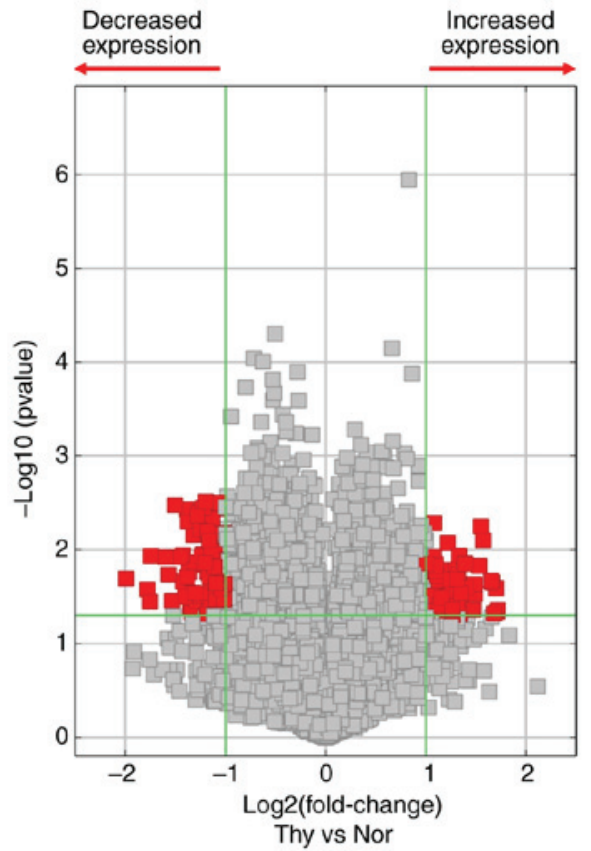

D

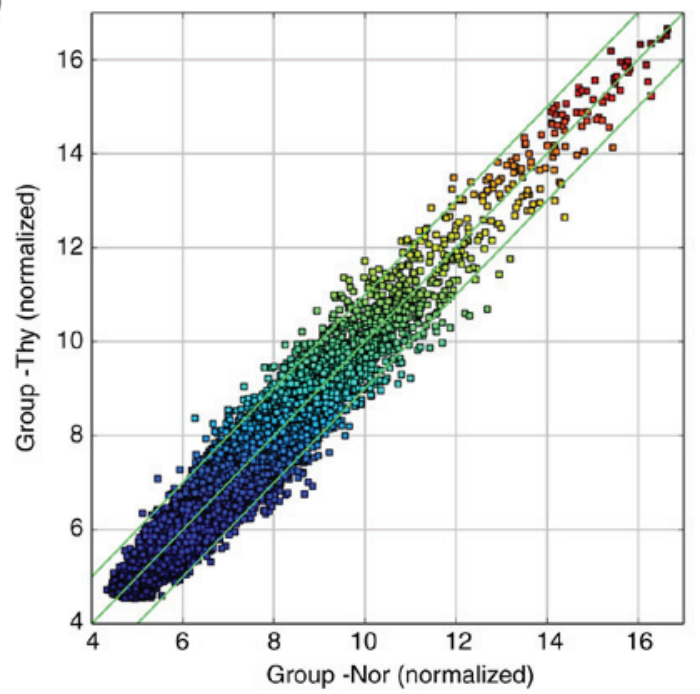

Figure 2. Difference analysis of circRNA microarray between PTC tumors and the adjacent tissues. (A) Heatmap of dysregulated circRNAs in two groups. Red represents high expression, while green represents low expression. (B) The volcano plot shows the differential expression of circRNAs in two groups. Red points indicated circRNAs expressed more/less than 2-fold change in PTC patients $(\mathrm{P}<0.05)$. (C) The box plot shows the stable distributions between samples. The distributions were nearly the same after normalization. (D) Scatter plots were used to identify differentially expressed circRNAs in two groups. The circRNAs above the top green line and below the bottom green line indicated more than a 2.0-fold change of circRNAs between two compared samples. $\mathrm{n}=3$ pairs of samples. Comparisons of data were acquired by the Student's t-test. circRNA, circular RNA; PTC, papillary thyroid cancer; Thy, PTC tumor tissues. Nor, PTC tumor-adjacent tissues.

were further annotated by KOBAS 3.0, which were enriched in a variety of pathways and diseases. The top 10 items of KEGG pathway and diseases are presented in Fig. 6B, including 'MAPK signaling pathway', 'PI3K-AKT signaling pathway', 'TNF signaling pathway', 'Olfactory transduction', 'Salmonella infection', 'Relaxin signaling parhway', 'Acute myeloid leukemia', 'Fcepsilon RI signaling pathway', 'Shigellosis', 'Bacterial invasion of epithelial cells' and 'cancers of endocrine organs', 'cancer', 'endocrine, metabolic diseases', respectively, indicating the role of hsa_circ_IPCEF1 in PTC regulation (Fig. 6B).

Based on a comprehensive search of other studies in human malignancies, it was found that four ceRNAs (CASR, CDC25B, NFkB1 and SHOC2) were cancer-related target genes, which were reported to be regulated by 21 miRNAs (hsa-miR-16-5p, hsa-miR-195-5p, hsa-miR-15a-5p, hsa-miR-6838-5p, hsa-miR-15b-5p, hsa-miR-497-5p, hsa-miR-424-5p, hsa-miR-4530, hsa-miR-1323, hsa-miR-3691-5p, hsa-miR-545-3p, hsa-miR-128-3p, hsa-miR-922, hsa-miR-3918, hsa-miR-3619-5p, hsa-miR-608, hsa-miR-1262, hsa-miR-761, hsa-miR-214-3p, hsa-miR-4651 and hsa-miR-370-3p, data not shown). Therefore, the circRNA-miRNA-mRNA module containing four mRNAs and 21 miRNAs was constructed in Cytoscape software to concentrate the ceRNA targets (Fig. 6C).

Among these miRNAs, hsa-miR-3619-5p, reported as a tumor inhibitor, was one of the top MREs of hsa_circ_IPCEF1, with two seed sequence matching regions (44). Bioinformatics 


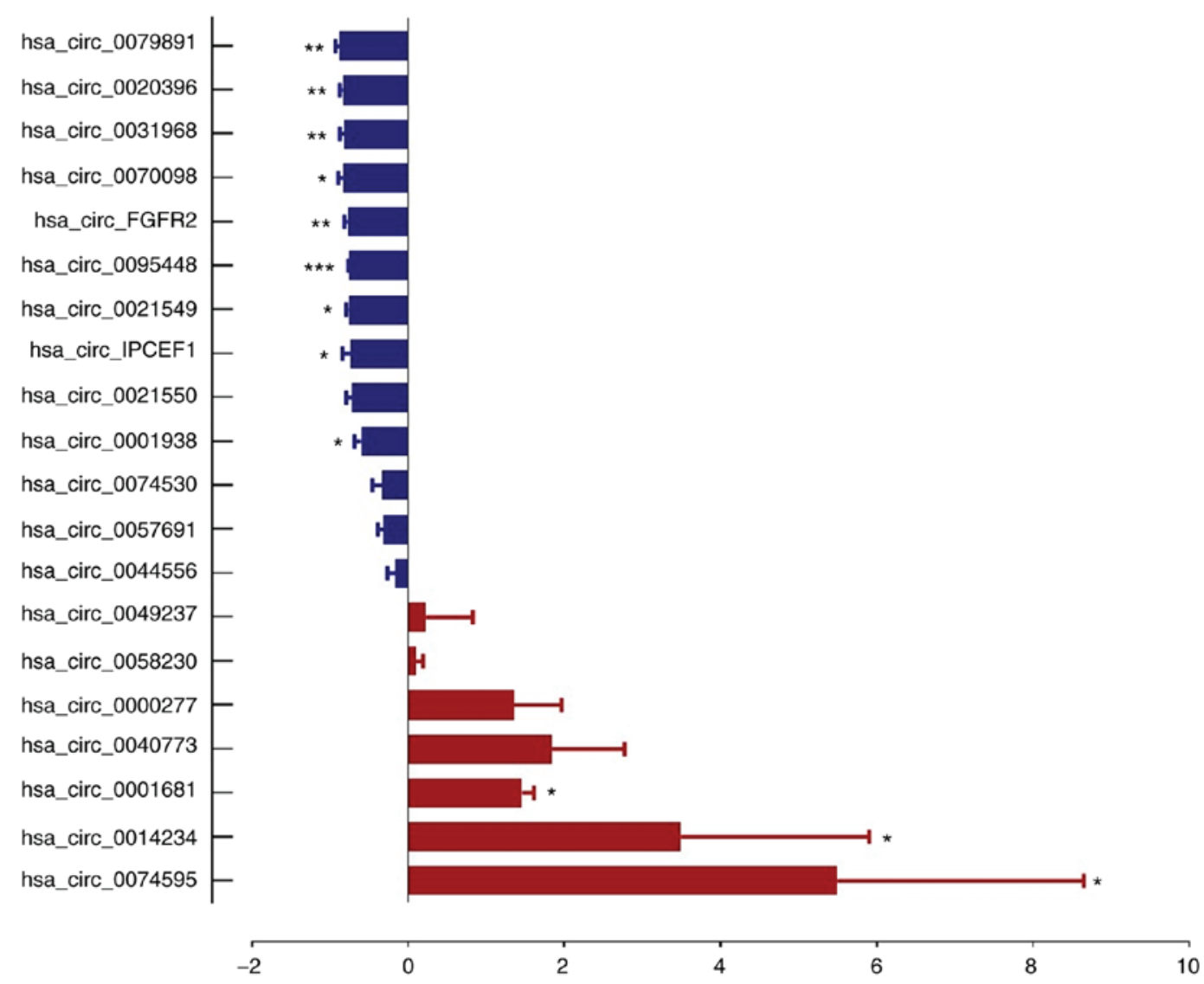

Figure 3. Confirmation of the differential expression of circRNAs in PTC tissues by reverse transcription-quantitative PCR. The relative expressions of 12 specific circRNAs between PTC and adjacent tissues were validated. ${ }^{*} \mathrm{P}<0.05,{ }^{* * *} \mathrm{P}<0.01,{ }^{* * * *} \mathrm{P}<0.001, \mathrm{n}=3$ pairs of samples. Comparisons of data were acquired by the Student's t-test. circRNA, circular RNA; PTC, papillary thyroid cancer.

A

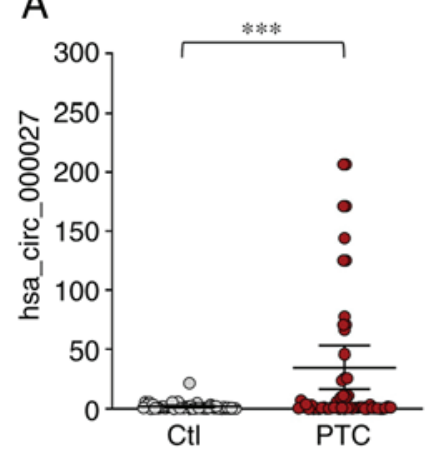

B

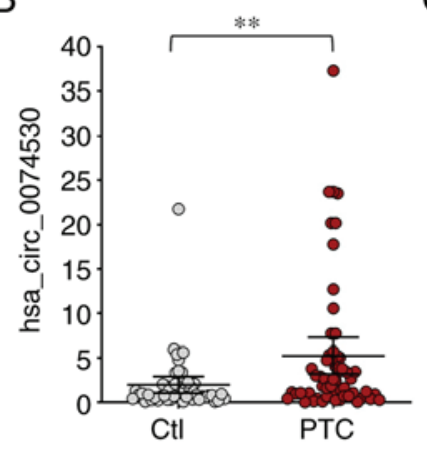

C

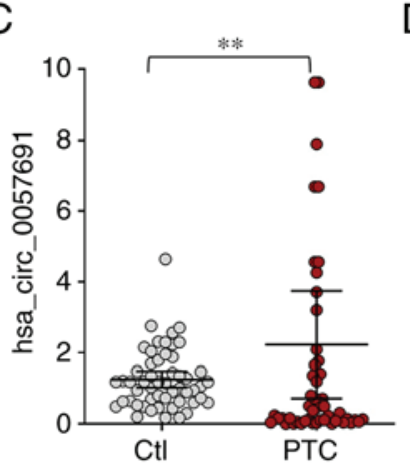

$\mathrm{D}$

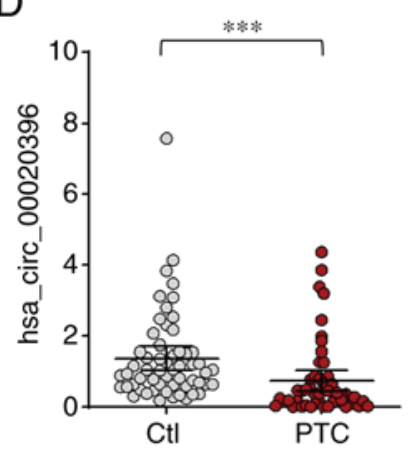

E

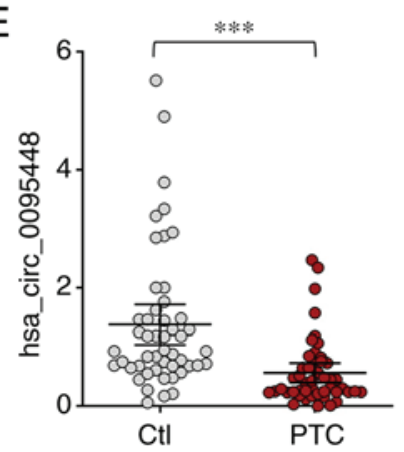

$\mathrm{F}$

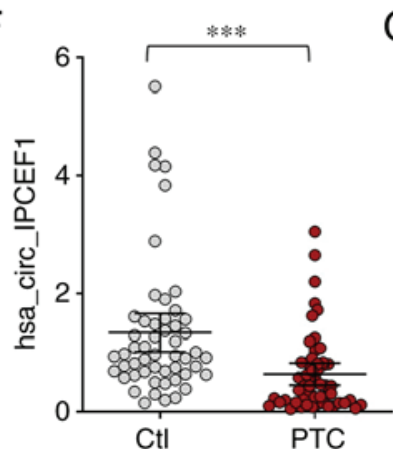

G

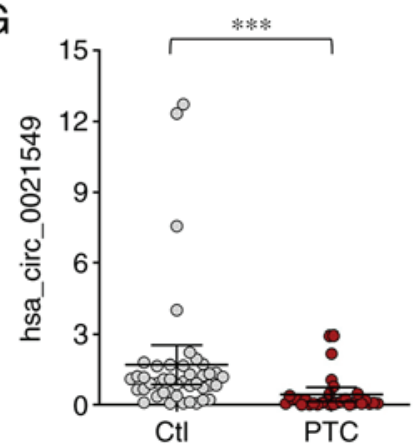

Figure 4. Confirmation of the differential expression of circRNAs in whole blood from PTC patients and healthy controls. (A) hsa_circ_0000277, (B) hsa circ_0074530, (C) hsa_circ_0057691 were significantly upregulated, while (D) hsa_circ_0020396, (E) hsa_circ_0095448, (F) hsa_circ_IPCEF1 and (G) hsa_circ_0021549 were significantly downregulated in PTC patients. ${ }^{* *} \mathrm{P}<0.01,{ }^{* * *} \mathrm{P}<0.001, \mathrm{n}=57$ pairs of subjects. Comparisons of data were acquired by one-way ANOVA followed by Bonferroni post hoc. circRNA/circ, circular RNA; PTC, papillary thyroid cancer; Ctl, healthy controls. 


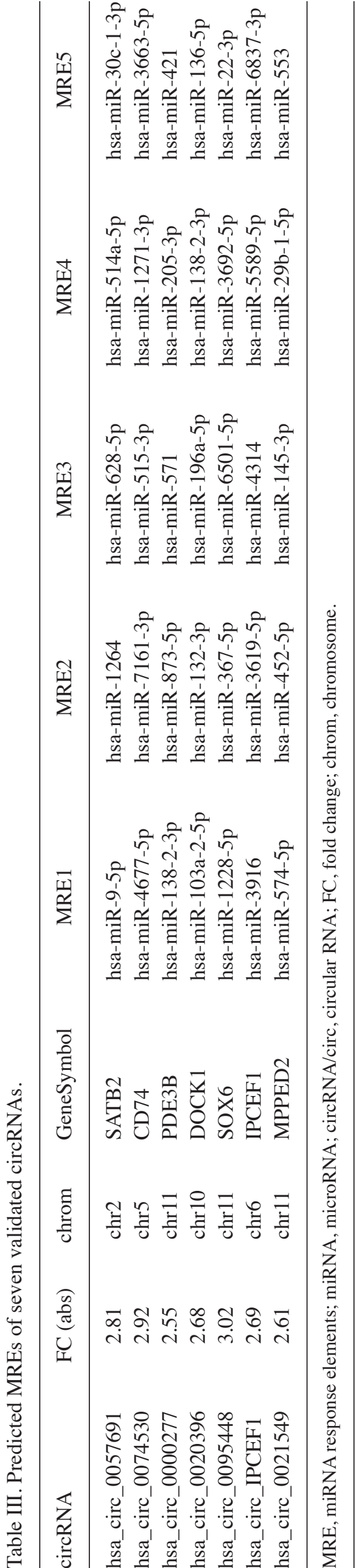

screening suggested that CDC25B (NM_021873) and CASR (NM_000388) were potential direct target genes of hsa-miR-3619-5p, with complementary binding sites located in the 3'-UTRs of CDC25B and CASR mRNAs, respectively (Fig. 7). These bioinformatics analyses indicated that hsa circ_IPCEF1/hsa-miR-3619-5p/target genes may be involved in PTC pathogenesis.

Prediction value of dysregulated circRNAs. To assess the performance of these seven circRNAs in predicting their potential association with PTC, their ROC curves were calculated and the accuracy evaluated with the areas under the ROC curves (AUCs). The preferable AUC value for circRNAs were hsa_circ_0021549 (AUC: 0.8194; 95\% CI: 0.7117 to $0.9270 ; \mathrm{P}<0.0001$ ) and hsa_circ_IPCEF1 (AUC: $0.801095 \%$ CI: 0.7108 to 0.8912 ; $P<0.0001$; Fig. $8 \mathrm{~A}-\mathrm{G}$ ). Instead of other circRNAs, hsa_circ_IPCEF1 exhibited the strongest functional role in ceRNA analysis, therefore, the correlation between hsa_circ_IPCEF1 expression and clinicopathological characteristics was examined in 57 patients with PTC. Statistical analysis showed that the downregulation of hsa_circ_IPCEF1 expression was associated with tumor lymph node metastasis in patients with PTC, suggesting its clinical value for PTC diagnosis and prognosis (Table I). To further confirm the formation of hsa_circ_IPCEF1 in patients with PTC, the splicing junction of hsa_circ_IPCEF1 was validated using RT-PCR and sequencing methods. As shown in Fig. 8H, hsa_circ_IPCEF1 is generated from the IPCEF1 gene located on human chromosome 6 (154520801-154544377). The 245bp products of hsa_circ_IPCEF1 in whole blood from subjects were amplified using divergent primers (Fig. 8I). Sanger sequencing further confirmed that the head-to-tail junction sites (AG/GC) were consistent with the hsa_circ_IPCEF1 annotation (Fig. 8J).

\section{Discussion}

circRNAs, a special novel class of endogenous non-coding RNAs, have been documented to serve crucial roles in various diseases by regulating numerous cellular events (45). Although numerous studies have focused on circRNAs as cancer markers, the diagnostic ability and understanding of circRNAs in PTC are still unclear. Therefore, the present study aimed to profile the expression levels of all circRNAs in patients with PTC, determine their potential roles in PTC and explore new insights into the pathogenesis of TC.

From microarray analysis, an aberrant expression pattern of circRNAs in PTC tissues compared to benign thyroid tissues was found. In liquid biopsy, the usage of whole blood is the main source of body fluid and circRNAs in blood cells or whole blood have been proposed as biomarkers for human diseases due to their high stability, abundance and spatiotemporal specific expression (46). Therefore, three upregulated and four downregulated circRNAs were verified in whole blood from 57 pairs of patients with PTC compared to healthy subjects and validated by RT-qPCR.

PTC originates from thyroid follicular epithelial cells, accounting for $89.8 \%$ of differentiated thyroid carcinomas, while only $38 \%$ of thyroid cancers show clinical symptoms (47). 
A

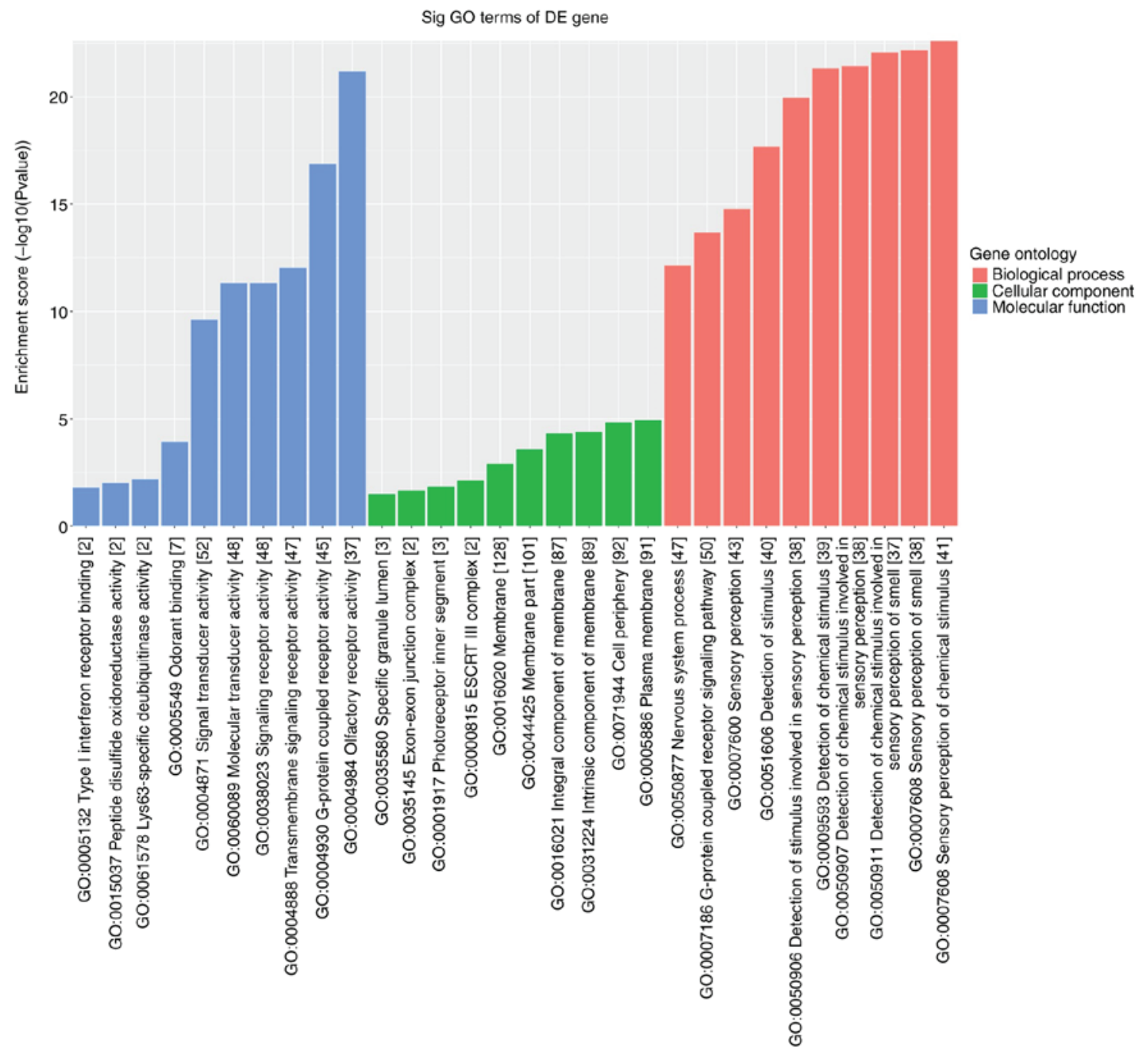

B

Sig pathway of DE gene

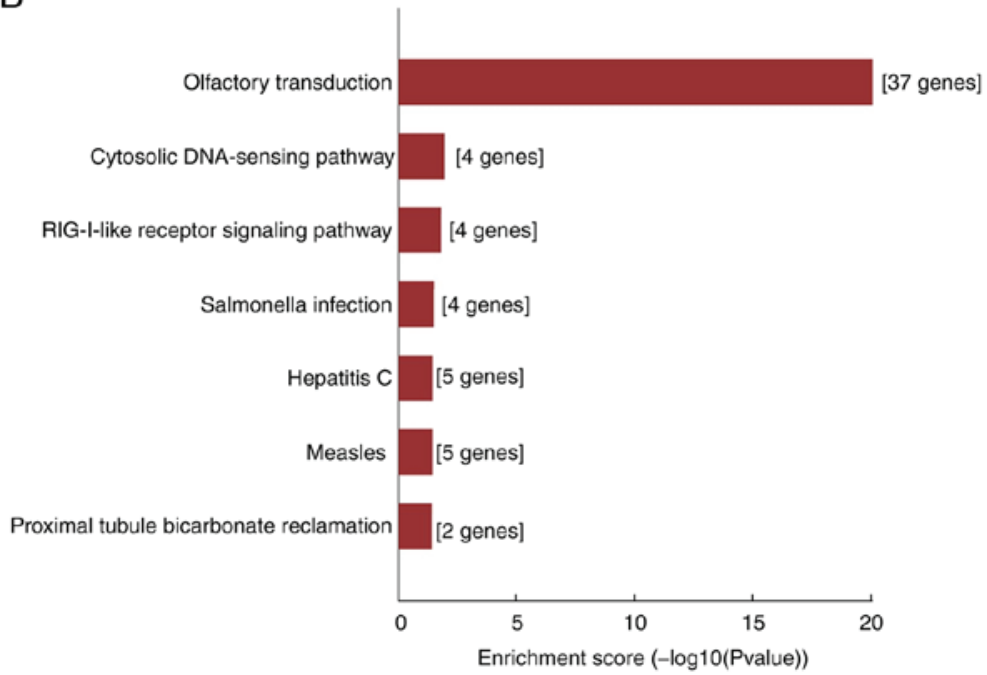

Figure 5. GO and KEGG analysis of the target genes of seven validated dysregulated circRNAs. (A) GO includes three domains: Biological Process, Cellular Component and Molecular Function. The P-value produced by top GO indicates the significance of GO terms abundance in seven dysregulated circRNAs targeting genes. (B) The seven pathways of seven validated dysregulated circRNAs targeting genes were identified using KEGG analysis according to the P-value. GO, Gene Oncology; KEGG, Kyoto Encyclopedia of Genes and Genomes; circRNA, circular RNA.

However, the extensive implementation of cancer imaging studies in preoperative diagnostics has resulted in a growing incidence of low-risk PTCs, which has caused a continuing debate about the adequacy of aggressive surgical approaches and radioiodine therapy (48). The molecular predictors of preoperative diagnostics and accurate treatment for PTC are 

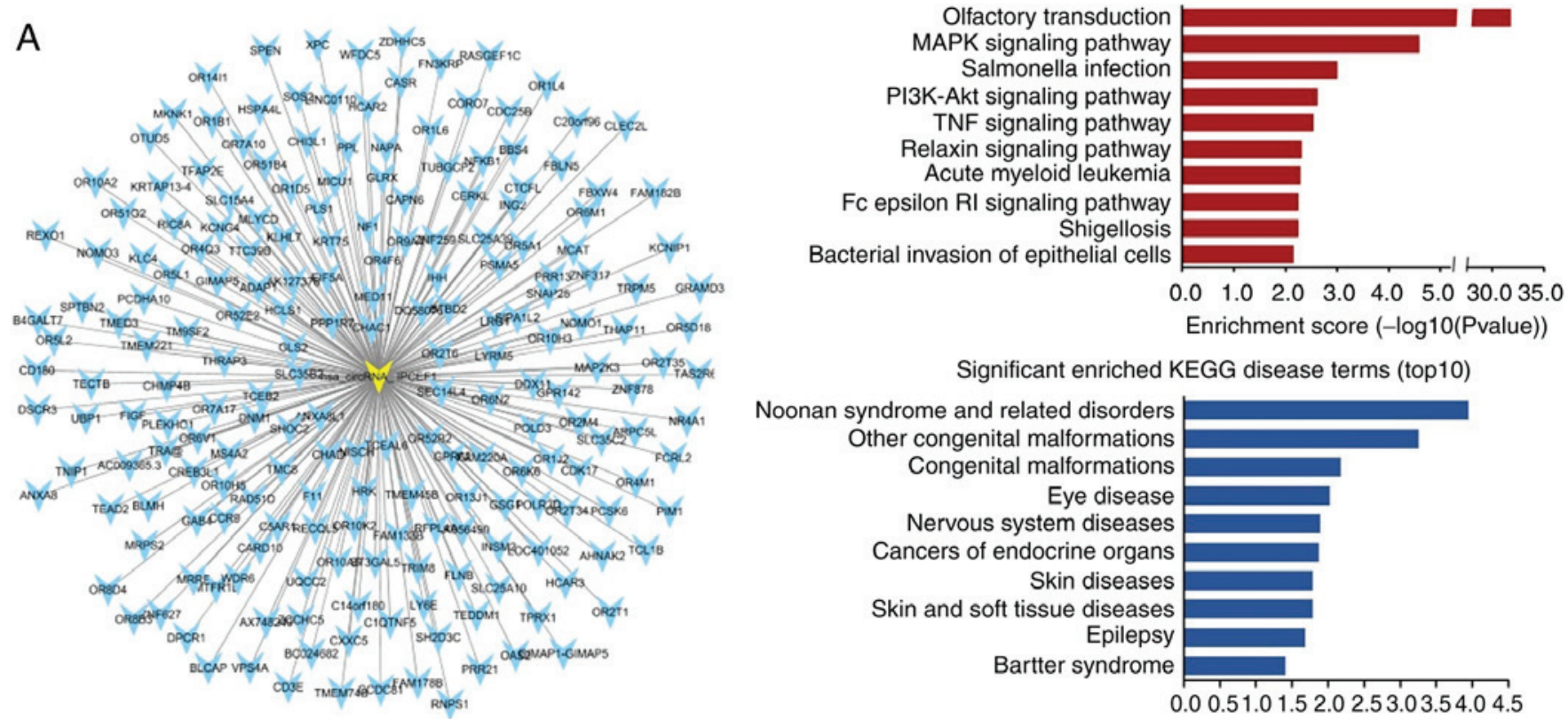

Significant enriched KEGG disease terms (top10)
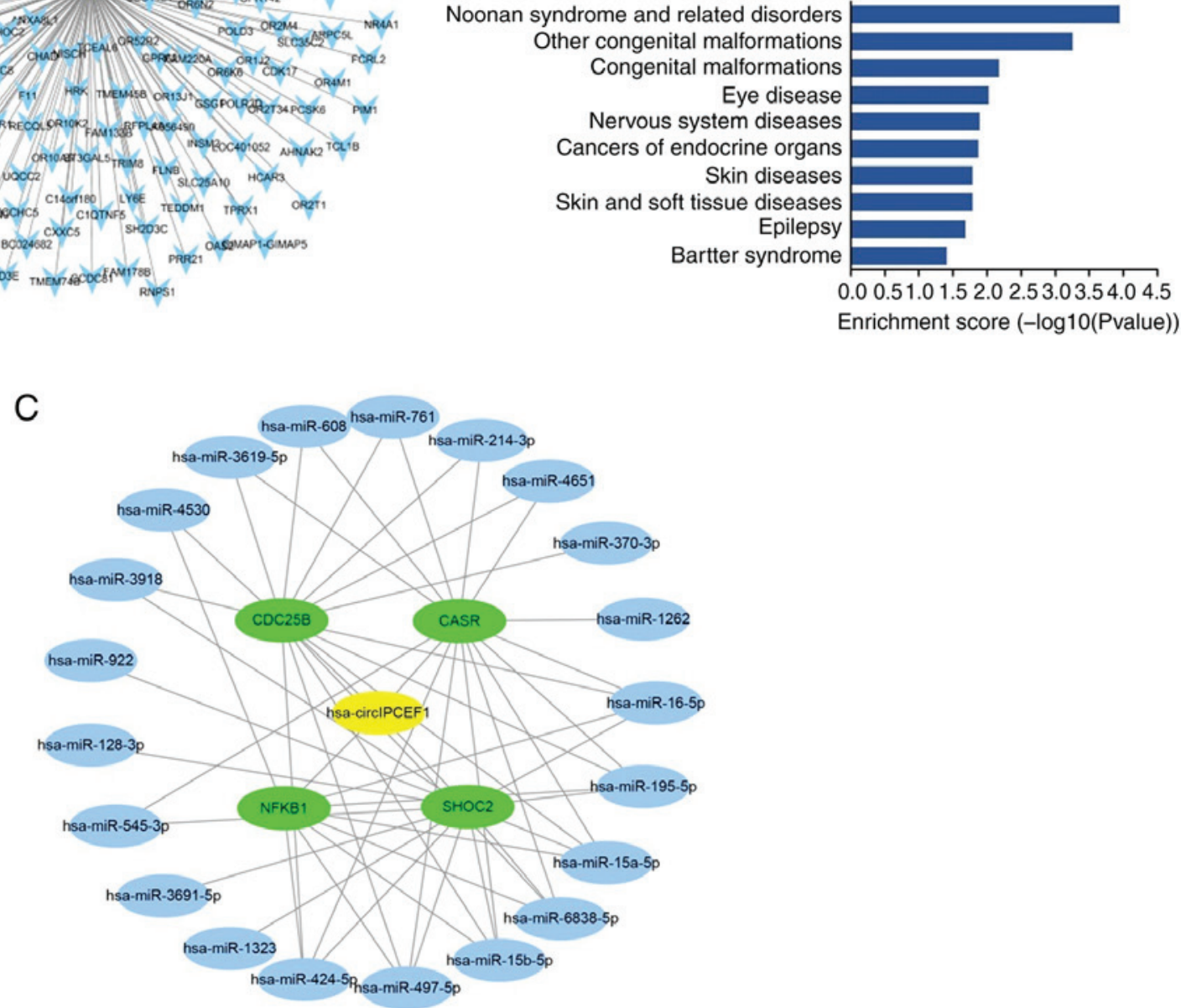

Figure 6. ceRNA analysis of hsa_circ_IPCEF1. (A) All 207 predicted ceRNA genes of hsa_circ_IPCEF1. (B) The top 10 enriched KEGG pathways and diseases of hsa_circ_IPCEF1 related ceRNAs. (C) The ceRNA network of hsa_circ_IPCEF1, which contains hsa_circ_IPCEF1 (represented by yellow node), 21 miRNAs (represented by blue nodes) and four target gene mRNAs related to thyroid cancer (represented by green nodes). ceRNA, competing endogenous RNA; KEGG, Kyoto Encyclopedia of Genes and Genomes; circ, circular RNA.

poorly defined. Although studies have focused on mRNA transcripts and non-coding RNAs as potential PTC markers, the expression profiles and roles of circRNAs in PTC remain to be elucidated (49-52). By contrast with recent studies in circRNA screening in PTC tissues, the present study validated seven dysregulated circRNAs (three upregulated and four downregulated) in whole blood from PTC subjects, which were found to be more suitable for biomarker detection in the future $(53,54)$. To explore the mechanisms of these dysregulated circRNAs on the pathological processes of PTC, GO and KEGG pathway analyses were performed. KEGG pathway analysis demonstrated that the olfactory transduction pathway was the most significant pathway for enrichment, followed by the cytosolic DNA-sensing pathway and the RIG-I-like receptor signaling pathway. Moreover, according to the BP, CC and MF terms with substantial enrichment, the genes were mainly associated with 'G-protein-coupled receptor activity', 'Signal receptor activity in the plasma membrane' and 'Olfactory receptor activity'. These results suggested that the deregulated circRNAs may participate in the pathological process of PTC. Recently, circRNAs have been well described as ceRNAs in human diseases, as has the possibility of using circRNAs as molecular markers for related diseases (55). Compared with miRNAs, circRNAs act on miRNA sponges to attenuate the 
A

circ_IPCEF1

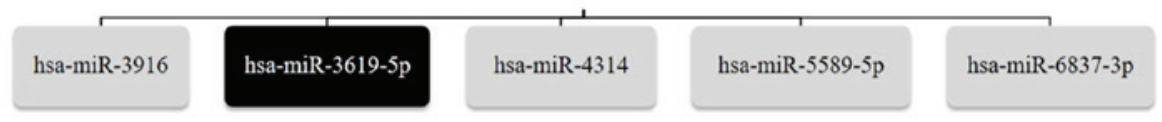

B

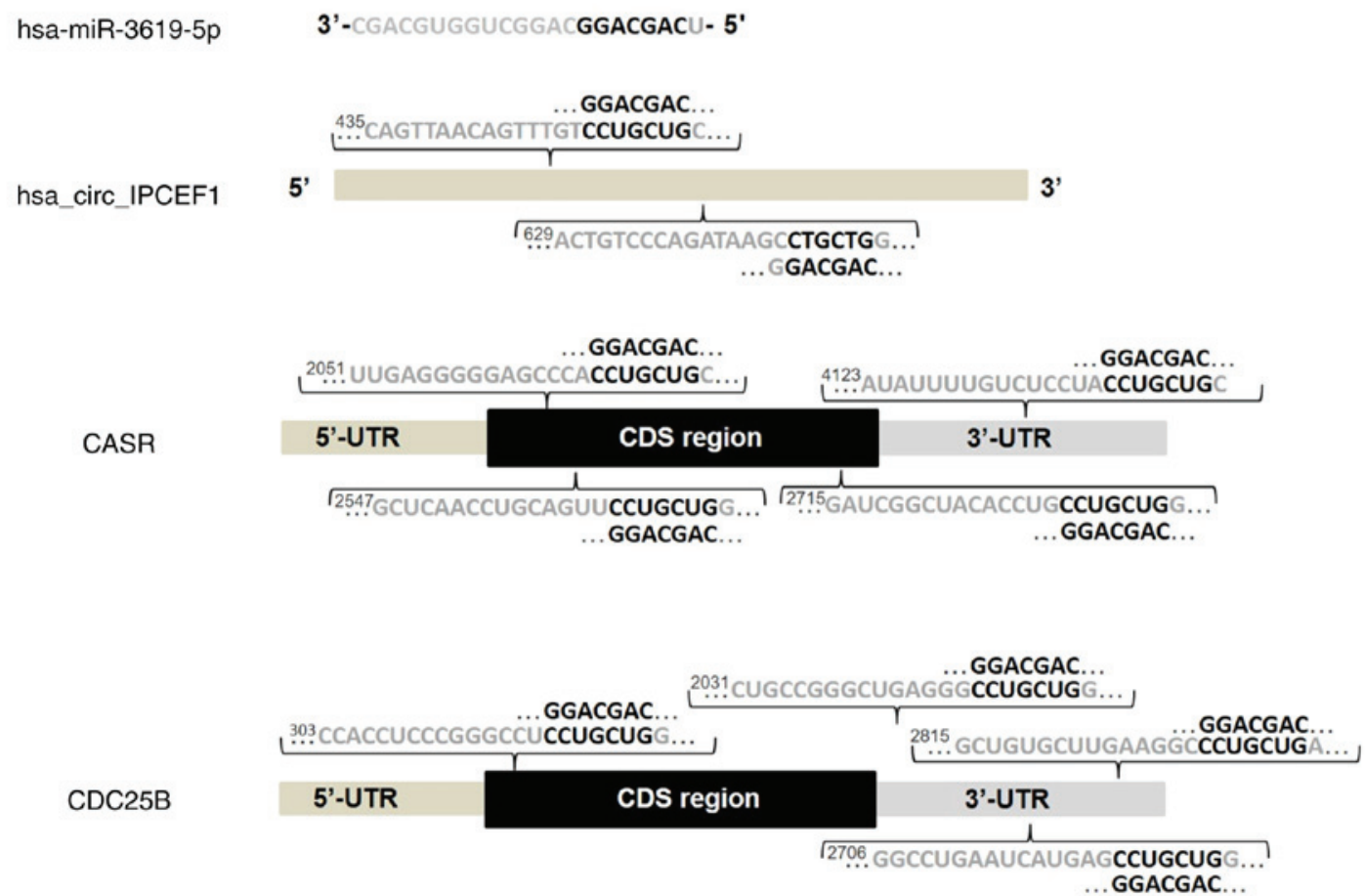

Figure 7. hsa_circ_IPCEF1, miRNAs and target genes interactions. (A) Predicted top five miRNAs for hsa_circ_IPCEF1 by ceRNA analysis. (B) Seed match regions of hsa_circ_IPCEF1, hsa_miR_3619-5p and target genes. miRNA, microRNA; ceRNA, competing endogenous RNA; circ, circular RNA.

effect of miRNAs on mRNA expression (42). For example, circCRIM1 promotes the expression of BTG2 by inhibiting the expression of miR-125b-5p in LUAC cells, thus affecting the growth of LUAC cells (37). The loss of circ-znf609 inhibits the proliferation and cell cycle transition and induces the apoptosis of NPC cells via modulation of the miR-188/elf2 axis (35). In bladder cancer, circRNA-cTFRC is upregulated and correlated with tumor grade and survival rate, acting as a miR-107 sponge to regulate TFRC expression, cancer cell invasion, proliferation, epithelial to mesenchymal transition and tumor growth (56). These findings suggest the key roles of abnormal circRNAs and target genes in the pathogenesis of disease, indicating a new perspective for the early diagnosis, treatment and prognosis of PTC.

Using a set of bioinformatics tools, the present study predicted MREs for the seven validated circRNAs and the potential targets of miRNAs. The present study found one promising downregulated circRNA (hsa_circ_IPCEF1) in PTC subjects, which was not indicated in any previously published literature but showed interactions with four cancer-related ceRNAs (CASR, CDC25B, NF- $\mathrm{B} 1$ and SHOC2). Notably, $\mathrm{NF}-\kappa \mathrm{B} 1$ is a transcription regulator that serves important roles in numerous biological processes, such as survival and proliferation, inflammation and adaptive immune responses and has been widely implicated in the development and progression of cancer pathogenesis (57-60). In cancer cells, chronic activation and nuclear localization result in $\mathrm{NF}-\kappa \mathrm{B} 1$ accumulation and $\mathrm{NF}-\kappa \mathrm{B}$ activation, which stimulate cell survival by inducing anti-apoptotic gene expression (61). Recently, $\mathrm{NF}-\kappa \mathrm{B} 1$ has been proved to be upregulated in a variety of cancers, including subungual keratoacanthoma, urothelial carcinoma and breast cancer, suggesting that it is a biomarker for diagnosis and treatment (62-66). In the results of the present study, $\mathrm{NF}-\kappa \mathrm{B} 1$ was predicted to be a ceRNA of hsa_circ_IPCEF1 regulation and the downregulation of hsa_circ_IPCEF1 in PTC was accompanied by the upregulation of $\mathrm{NF}-\kappa \mathrm{B} 1$, suggesting that a combination of hsa_circ_IPCEF1 and NF- $\kappa$ B1 could be used as biomarkers for PTC diagnosis/prognosis. The ROC analyses of RT-qPCR results, as well as correlation analysis between hsa_circ_IPCEF1 and clinical parameters, further confirmed hsa_circ_IPCEF1 as one preferable candidate biomarker for PTC. Moreover, the circRNA-miRNA-mRNA module suggested that hsa-miR-3619-5p was a direct target for hsa_circ_IPCEF1 sponging. As a tumor suppressor, hsa-miR-3619-5p inhibits prostate cancer cell growth and hampers the proliferation of cutaneous squamous cell carcinoma and liver cancer cells, exerting tumor inhibitory effects on human malignancies (67-69). Bioinformatics analysis further confirmed the interactions between hsa-miR-3619-5p and CDC25B (an initiator of mitosis) and CASR (calcium-sensing receptor) mRNAs, which may be direct targets of hsa_circ IPCEF1/hsa-miR-3619-5p axis regulation. By controlling the 
A

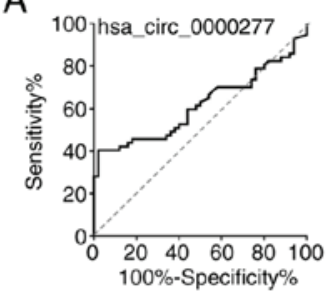

B

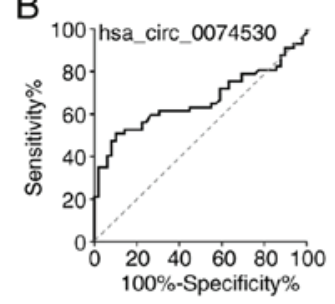

C

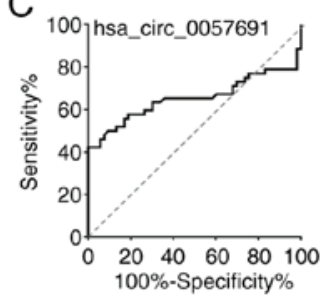

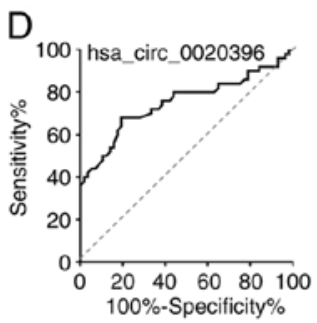

$\mathrm{E}$

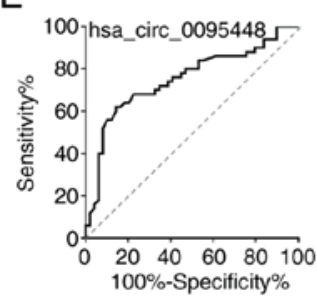

$\mathrm{F}$

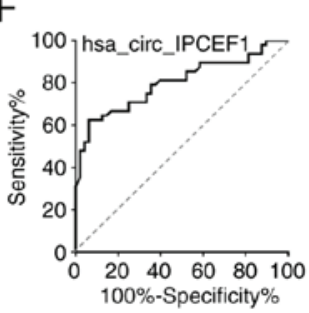

G
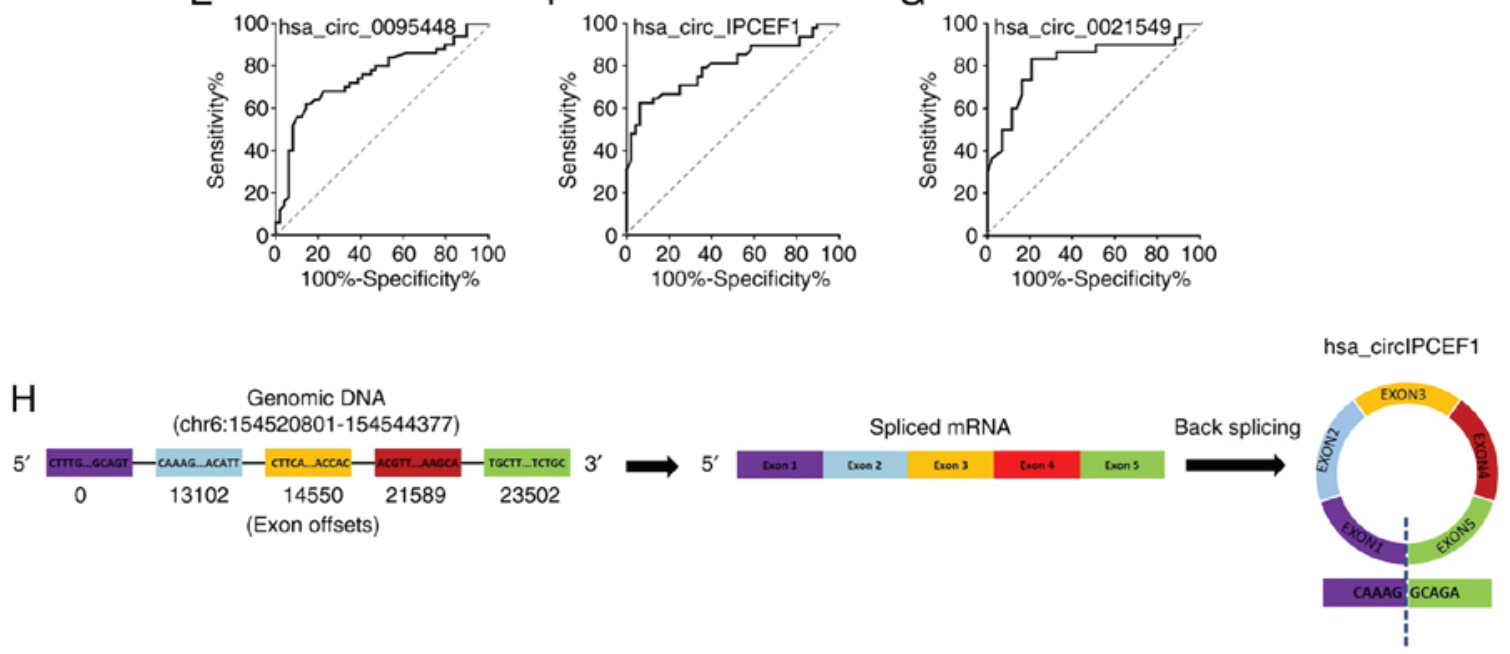

I

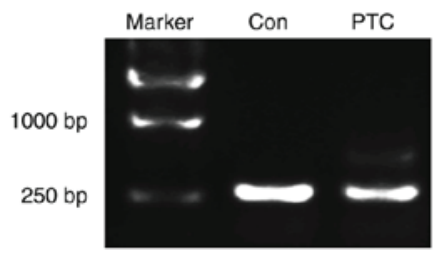

$J$

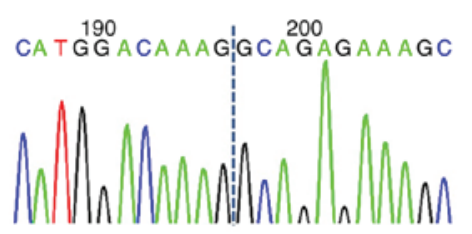

Figure 8. Evaluation candidate circRNAs. (A-G) Receiver-operating characteristic curve analysis of seven circRNAs in 57 paired whole blood of PTC patients. $\mathrm{n}=57$ pairs of subjects. Comparisons of data were acquired by one-way ANOVA followed by Bonferroni post hoc. Identification of hsa_circ_IPCEF1 circularization. (H) Annotation of hsa_circ_IPCEF1. (I) Reverse transcription PCR and (J) sequencing analysis of head-to-tail splicing junction of hsa_circ_IPCEF1 in whole blood from PTC and healthy subjects. circRNA/circ, circular RNA; PTC, papillary thyroid cancer.

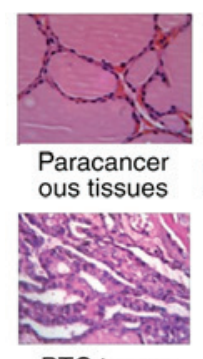

PTC tumor

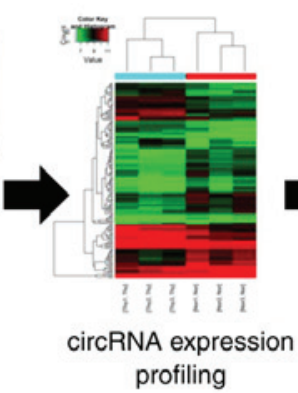

profiling
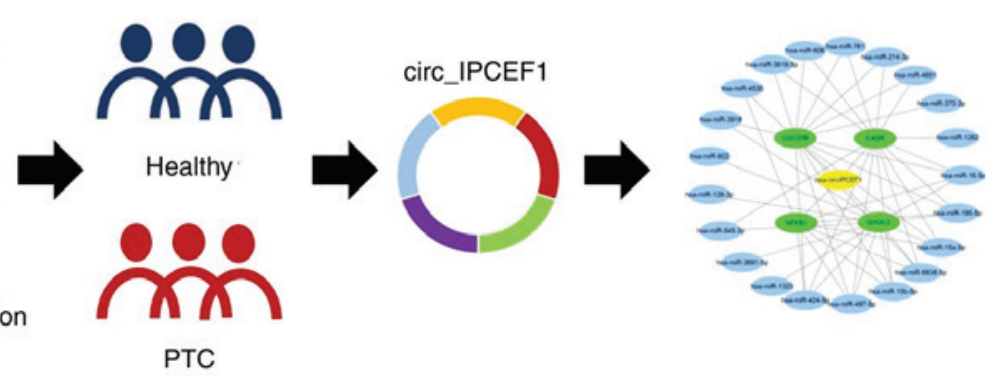

Figure 9. Schematic diagram of the proposed role of hsa_circ_IPCEF1 in PTC. PTC, papillary thyroid cancer; circRNA, circular RNA.

$\mathrm{G}_{2} / \mathrm{M}$ cell cycle phase transition and calcium metabolism, $\mathrm{CDC} 25 \mathrm{~B}$ and CASR serve important roles in the occurrence and metastasis of cancer, respectively. These results suggested that hsa_circ_IPCEF1 may participate in the pathological process of PTC through sponging hsa-miR-3619-5p. Clearly, the functions and mechanisms of the hsa_circ_IPCEF1/miR-3619-5p axis need to be further investigated in PTC cells to conclusively determine which target genes interact which cellular functions are affected during tumorigenesis. Taken together, the present study identified distinctive circRNA expression patterns in PTC. hsa_circ_IPCEF1 may bind to hsa-miR-3619-5p through sponge action, thus regulating the target genes of cell proliferation, migration and invasion and triggering the initiation and progression of PTC. These results provide insight into the pathogenesis of PTC and suggest the potential roles of hsa_circ_IPCEF1 in PTC diagnosis and treatment. 
There are still some potential limitations and shortcomings in the present study. First, in PTC diagnosis, diagnostic performance was primarily based on neck ultrasonic diagnosis rather than expensive fine-needle aspiration cytology. PTC tumors were obtained from aggressive surgical approaches on patients with high-risk PTC, who were diagnosed and evaluated by neck ultrasonic imaging and immunohistochemistry. Limited by the small size of PTC tumors, the present study did not perform diagnostic assays on these samples to clarify the PTC subtype and genotype. However, the PTC tumors obtained for microarray were conventional PTC, exhibiting fibrovascular cores and enlarged nuclei with nuclear clearing, which were sufficiently accurate for the present study. Second, the correlation between the expression level of hsa_circ_IPCEF1 and the clinical parameters in patients with PTC was analyzed only in 57 pairs of subjects. A new set of patients with PTC should be recruited to test the predictive value and the utility of hsa_circ_IPCEF1 as a prognostic biomarker in the near future. Nevertheless, the present study provided new insight into the pathogenesis of PTC at the molecular level and proposes one potential candidate PTC biomarker. Third, the analysis of the present study was limited to the tissue and whole blood of patients with PTC and the biological function of the maladjusted circRNAs was predicted only through bioinformatics. The effects of the dysregulated circRNAs on the proliferation and migration of PTC tumor cells still need to be studied in vivo and in vitro. Also, the construction of the ceRNA network is based on the sponge function of circRNAs; other regulatory functions still need to be further studied. The mechanisms and functions of the hsa_circ_IPCEF1/hsa-miR-3619-5p axis in PTC will be studied in the near future.

In conclusion, the present study reported seven significantly differentiated circRNAs in patients with PTC compared to healthy subjects, suggesting a key role of dysregulated circRNAs in the pathogenesis of PTC. As a downregulated circRNA, hsa_circ_IPCEF1 may show promise as a potential biomarker for PTC. Moreover, the bioinformatics analysis suggested that the hsa_circ_IPCEF1/hsa-miR-3619-5p axis may be involved in the pathogenesis of PTC, providing a new theoretical basis for the future diagnosis and treatment of PTC (Fig. 9).

\section{Acknowledgements}

Not applicable.

\section{Funding}

This study was supported by the National Natural Science Foundation of China (Contract grant no. 81770054); National Natural Science Foundation of China (Contract grant no. 8197052197); Chinese Natural science foundation of Heilongjiang province (grant no. LH2019H017); Tianjin Municipal Science and Technology Project (grant no. 18JCZDJC44900); The Fundamental Research Funds for the Central Universities-Nankai University (grant no. 63191150); Spark Research Fund from The Fourth Affiliated Hospital of Harbin Medical University (grant no. HYDSYXH201907).

\section{Availability of data and materials}

The datasets used and/or analyzed during the current study are available from the corresponding author on reasonable request.

\section{Authors' contributions}

SSL and XJ designed the study and interpreted the data. MG analyzed and interpreted the patient data regarding papillary thyroid carcinoma. SSL and YS performed the histological examination of PTC, molecular studies and were major contributors in writing the manuscript. JD, YL and SY performed RT-qPCR experiments. YZ and XJ performed bioinformatics analysis. SSL and XJ confirm the authenticity of all the raw data. All authors read and approved the final manuscript.

\section{Ethics approval and consent to participate}

The study procedure was approved by Medical Ethics Committee of Harbin Medical University (approval no. IRB3011619).

\section{Patient consent for publication}

Not applicable.

\section{Competing interests}

The authors declare that they have no competing interests.

\section{References}

1. Kilfoy BA, Zheng T, Holford TR, Han X, Ward MH, Sjodin A, Zhang Y, Bai Y, Zhu C, Guo GL, et al: International patterns and trends in thyroid cancer incidence, 1973-2002. Cancer Causes Control 20: 525-531, 2009.

2. Cai X, Zhao Z, Dong J, Lv Q, Yun B, Liu J, Shen Y, Kang J and Li J: Circular RNA circBACH2 plays a role in papillary thyroid carcinoma by sponging miR-139-5p and regulating LMO4 expression. Cell Death Dis 10: 184, 2019.

3. Heidari Z, Mohammadpour-Gharehbagh A, Eskandari M, Harati-Sadegh $M$ and Salimi S: Genetic polymorphisms of miRNA let7a-2 and pri-mir-34b/c are associated with an increased risk of papillary thyroid carcinoma and clinical/pathological features. J Cell Biochem: Dec 14, 2018 (Epub ahead of print). doi: $10.1002 /$ jcb.28152.

4. La Vecchia C, Malvezzi M, Bosetti C, Garavello W, Bertuccio P, Levi F and Negri E: Thyroid cancer mortality and incidence: A global overview. Int J Cancer 136: 2187-2195, 2015.

5. Akslen LA, Haldorsen T, Thoresen SO and Glattre E: Incidence pattern of thyroid cancer in Norway: Influence of birth cohort and time period. Int J Cancer 53: 183-187, 1993.

6. Colonna M, Grosclaude P, Remontet L, Schvartz C, Mace-Lesech J, Velten M, Guizard A, Tretarre B, Buemi AV, Arveux $\mathrm{P}$ and Esteve $\mathrm{J}$ : Incidence of thyroid cancer in adults recorded by French cancer registries (1978-1997). Eur J Cancer 38: 1762-1768, 2002.

7. dos Santos Silva I and Swerdlow AJ: Thyroid cancer epidemiology in England and Wales: Time trends and geographical distribution. Br J Cancer 67: 330-340, 1993.

8. Montanaro F, Pury P, Bordoni A and Lutz JM; Swiss Cancer Registries Network: Unexpected additional increase in the incidence of thyroid cancer among a recent birth cohort in Switzerland. Eur J Cancer Prev 15: 178-186, 2006.

9. Pettersson B, Adami HO, Wilander E and Coleman MP: Trends in thyroid cancer incidence in Sweden, 1958-1981, by histopathologic type. Int J Cancer 48: 28-33, 1991. 
10. Ferlay J, Colombet M and Bray F: Cancer incidence in five continents, CI5plus: IARC CancerBase No. 9 [Internet]. Lyon, France: International Agency for Research on Cancer, 2018.

11. Pathak KA, Leslie WD, Klonisch TC and Nason RW: The changing face of thyroid cancer in a population-based cohort. Cancer Med 2: 537-544, 2013.

12. Jayarangaiah A, Sidhu G, Brown J, Barrett-Campbell O, Bahtiyar G, Youssef I, Arora S, Skwiersky S and McFarlane SI: Therapeutic options for advanced thyroid cancer. Int J Clin Endocrinol Metab 5: 26-34, 2019.

13. Rahmani N, Abbas Hashemi S, Fazli M and Raisian M: Clinical management and outcomes of papillary, follicular and medullary thyroid cancer surgery. Med Glas (Zenica) 10: 164-167, 2013.

14. Lin JD, Chao TC, Huang MJ, Weng HF and Tzen KY: Use of radioactive iodine for thyroid remnant ablation in well-differentiated thyroid carcinoma to replace thyroid reoperation. Am J Clin Oncol 21: 77-81, 1998.

15. Gottlieb JA and Hill CS Jr: Chemotherapy of thyroid cancer with adriamycin. Experience with 30 patients. N Engl J Med 290: 193-197, 1974

16. Long MY, Chen JW, Zhu Y, Luo DY, Lin SJ, Peng XZ, Tan LP and Li HH: Comprehensive circular RNA profiling reveals the regulatory role of circRNA_0007694 in papillary thyroid carcinoma. Am J Transl Res 12: 1362-1378, 2020.

17. Dong S, Xie XJ, Xia Q and Wu YJ: Indicators of multifocality in papillary thyroid carcinoma concurrent with Hashimoto's thyroiditis. Am J Cancer Res 9: 1786-1795, 2019

18. Xing M, Westra WH, Tufano RP, Cohen Y, Rosenbaum E Rhoden KJ, Carson KA, Vasko V, Larin A, Tallini G, et al: BRAF mutation predicts a poorer clinical prognosis for papillary thyroid cancer. J Clin Endocrinol Metab 90: 6373-6379, 2005

19. Basolo F, Torregrossa L, Giannini R, Miccoli M, Lupi C, Sensi E, Berti P, Elisei R, Vitti P, Baggiani A and Miccoli P: Correlation between the BRAF V600E mutation and tumor invasiveness in papillary thyroid carcinomas smaller than 20 millimeters: Analysis of 1060 cases. J Clin Endocrinol Metab 95: 4197-4205, 2010.

20. Elisei R, Viola D, Torregrossa L, Giannini R, Romei C, Ugolini C, Molinaro E, Agate L, Biagini A, Lupi C, et al: The $\mathrm{BRAF}(\mathrm{V} 600 \mathrm{E})$ mutation is an independent, poor prognostic factor for the outcome of patients with low-risk intrathyroid papillary thyroid carcinoma: Single-institution results from a large cohort study. J Clin Endocrinol Metab 97: 4390-4398, 2012.

21. Markopoulos GS, Roupakia E, Tokamani M, Chavdoula E, Hatziapostolou M, Polytarchou C, Marcu KB, Papavassiliou AG, Sandaltzopoulos R and Kolettas E: A step-by-step microRNA guide to cancer development and metastasis. Cell Oncol (Dordr) 40: 303-339, 2017.

22. Shukla GC and Gupta S: Hallmarks of cancer-focus on RNA metabolism and regulatory noncoding RNAs. Cancer Lett 420: 208-209, 2018

23. Calin GA, Dumitru CD, Shimizu M, Bichi R, Zupo S, Noch E, Aldler H, Rattan S, Keating M, Rai K, et al: Frequent deletions and down-regulation of micro-RNA genes miR 15 and miR16 at 13 q14 in chronic lymphocytic leukemia. Proc Natl Acad Sci USA 99: 15524-15529, 2002.

24. Fabbri M, Garzon R, Cimmino A, Liu Z, Zanesi N, Callegari E, Liu S, Alder H, Costinean S, Fernandez-Cymering C, et al: MicroRNA-29 family reverts aberrant methylation in lung cancer by targeting DNA methyltransferases $3 \mathrm{~A}$ and 3B. Proc Natl Acad Sci USA 104: 15805-15810, 2007.

25. Ferreira AF, Moura LG, Tojal I, Ambrósio L, Pinto-Simões B, Hamerschlak N, Calin GA, Ivan C, Covas DT, Kashima S and Castro FA: ApoptomiRs expression modulated by BCR-ABL is linked to CML progression and imatinib resistance. Blood Cells Mol Dis 53: 47-55, 2014.

26. Iorio MV and Croce CM: MicroRNA dysregulation in cancer: Diagnostics, monitoring and therapeutics. A comprehensive review. EMBO Mol Med 4: 143-159, 2012.

27. Scholl V, Hassan R and Zalcberg IR: miRNA-451: A putative predictor marker of Imatinib therapy response in chronic myeloid leukemia. Leuk Res 36: 119-121, 2012.

28. Muhammad N, Bhattacharya S, Steele R and Ray RB Anti-miR-203 suppresses ER-positive breast cancer growth and stemness by targeting SOCS3. Oncotarget 7: 58595-58605, 2016.

29. Cardona-Monzonís A, García-Giménez JL, Mena-Mollá S, Pareja-Galeano H, de la Guía-Galipienso F, Lippi G, Pallardó FV and Sanchis-Gomar F: Non-coding RNAs and coronary artery disease. Adv Exp Med Biol 1229: 273-285, 2020.
30. Zhang Y, Xue W, Li X, Zhang J, Chen S, Zhang JL, Yang L and Chen LL: The biogenesis of nascent circular RNAs. Cell Rep 15: 611-624, 2016

31. Kristensen LS, Andersen MS, Stagsted LVW, Ebbesen KK, Hansen TB and Kjems J: The biogenesis, biology and characterization of circular RNAs. Nat Rev Genet 20: 675-691, 2019

32. Memczak S, Jens M, Elefsinioti A, Torti F, Krueger J, Rybak A, Maier L, Mackowiak SD, Gregersen LH, Munschauer M, et al: Circular RNAs are a large class of animal RNAs with regulatory potency. Nature 495: 333-338, 2013.

33. Shan C, Zhang Y, Hao X, Gao J, Chen X and Wang K: Biogenesis, functions and clinical significance of circRNAs in gastric cancer. Mol Cancer 18: 136, 2019.

34. Kristensen LS, Hansen TB, Venø MT and Kjems J: Circular RNAs in cancer: Opportunities and challenges in the field. Oncogene 37: 555-565, 2018

35. Mingyan L, Yujie L and Min Y: CircRNA ZNF609 knockdown suppresses cell growth via modulating miR-188/ELF2 axis in nasopharyngeal carcinoma. Onco Targets Ther 13: 2399-2409, 2020.

36. Liang HF, Zhang XZ, Liu BG, Jia GT and Li WL: Circular RNA circ-ABCB10 promotes breast cancer proliferation and progression through sponging miR-1271. Am J Cancer Res 7: 1566-1576, 2017.

37. Zhang SJ, Ma J, Wu JC, Hao ZZ, Zhang YA and Zhang YJ: Circular RNA circCRIM1 suppresses lung adenocarcinoma cell migration, invasion, EMT, and glycolysis through regulating miR-125b-5p/BTG2 axis. Eur Rev Med Pharmacol Sci 24: 3761-3774, 2020

38. Livak KJ and Schmittgen TD: Analysis of relative gene expression data using real-time quantitative PCR and the 2(-Delta Delta $\mathrm{C}(\mathrm{T})$ ) method. Methods 25: 402-408, 2001

39. Garcia DM, Baek D, Shin C, Bell GW, Grimson A and Bartel DP: Weak seed-pairing stability and high target-site abundance decrease the proficiency of 1sy- 6 and other microRNAs. Nat Struct Mol Biol 18: 1139-1146, 2011.

40. Grimson A, Farh KK, Johnston WK, Garrett-Engele P, Lim LP and Bartel DP: MicroRNA targeting specificity in mammals: Determinants beyond seed pairing. Mol Cell 27: 91-105, 2007.

41. Enright AJ, John B, Gaul U, Tuschl T, Sander C and Marks DS: MicroRNA targets in Drosophila. Genome Biol 5: R1, 2003.

42. Salmena L, Poliseno L, Tay Y, Kats L and Pandolfi PP: A ceRNA hypothesis: The rosetta stone of a hidden RNA language? Cell 146: 353-358, 2011

43. Li JH, Liu S, Zhou H, Qu LH and Yang JH: starBase v2.0: Decoding miRNA-ceRNA, miRNA-ncRNA and protein-RNA interaction networks from large-scale CLIP-Seq data. Nucleic Acids Res 42 (Database Issue): D92-D97, 2014

44. Zhang Q, Miao S, Han X, Li C, Zhang M, Cui K, Xiong T, Chen Z, Wang C and Xu H: MicroRNA-3619-5p suppresses bladder carcinoma progression by directly targeting $\beta$-catenin and CDK2 and activating p21. Cell Death Dis 9: 960,2018.

45. Li Y, Fan H, Sun J, Ni M, Zhang L, Chen C, Hong X, Fang F, Zhang W and Ma P: Circular RNA expression profile of Alzheimer's disease and its clinical significance as biomarkers for the disease risk and progression. Int J Biochem Cell Biol 123: 105747,2020

46. Wen G, Zhou $\mathrm{T}$ and $\mathrm{Gu} \mathrm{W}$ : The potential of using blood circular RNA as liquid biopsy biomarker for human diseases. Protein Cell: Nov 1, 2020 (Epub ahead of print). doi: 10.1007/ s13238-020-00799-3.

47. Rahman ST, McLeod DSA, Pandeya N, Neale RE, Bain CJ, Baade P, Youl PH and Jordan SJ: Understanding pathways to the diagnosis of thyroid cancer: Are there ways we can reduce over-diagnosis? Thyroid 29: 341-348, 2019.

48. Krajewska J, Kukulska A, Oczko-Wojciechowska M, Kotecka-Blicharz A, Drosik-Rutowicz K, Haras-Gil M, Jarzab B and Handkiewicz-Junak D: Early diagnosis of low-risk papillary thyroid cancer results rather in overtreatment than a better survival. Front Endocrinol (Lausanne) 11: 571421, 2020

49. Bruland O, Fluge $\varnothing$, Akslen LA, Eiken HG, Lillehaug JR, Varhaug JE and Knappskog PM: Inverse correlation between PDGFC expression and lymphocyte infiltration in human papillary thyroid carcinomas. BMC Cancer 9: 425, 2009.

50. Stuchi LP, Castanhole-Nunes MMU, Maniezzo-Stuchi N Biselli-Chicote PM, Henrique T, Padovani Neto JA, de-Santi Neto D, Girol AP, Pavarino EC and Goloni-Bertollo EM: VEGFA and NFE2L2 gene expression and regulation by MicroRNAs in thyroid papillary cancer and colloid goiter. Genes (Basel) 11: 954, 2020. 
51. Lin X, Wu JF, Wang DM, Zhang J, Zhang WJ and Xue G: The correlation and role analysis of KCNK $2 / 4 / 5 / 15$ in human papillary thyroid carcinoma microenvironment. J Cancer 11: 5162-5176, 2020.

52. Gugnoni M, Manicardi V, Torricelli F, Sauta E, Bellazzi R, Manzotti G, Vitale E, de Biase D, Piana S and Ciarrocchi A: Linc00941 is a novel transforming growth factor $\beta$ target that primes papillary thyroid cancer metastatic behavior by regulating the expression of cadherin 6. Thyroid 31: 247-263, 2021.

53. Peng N, Shi L, Zhang Q, Hu Y, Wang N and Ye H: Microarray profiling of circular RNAs in human papillary thyroid carcinoma. PLoS One 12: e0170287, 2017.

54. Ren H, Liu Z, Liu S, Zhou X, Wang H, Xu J, Wang D and Yuan G: Profile and clinical implication of circular RNAs in human papillary thyroid carcinoma. PeerJ 6: e5363, 2018.

55. Qi X, Zhang DH, Wu N, Xiao JH, Wang X and Ma W: ceRNA in cancer: Possible functions and clinical implications. J Med Genet 52: 710-718, 2015.

56. Su H, Tao T, Yang Z, Kang X, Zhang X, Kang D, Wu S and Li C: Circular RNA cTFRC acts as the sponge of MicroRNA-107 to promote bladder carcinoma progression. Mol Cancer 18: 27, 2019.

57. Tang YQ, Jaganath IB, Manikam R and Sekaran SD: Inhibition of MAPKs, Myc/Max, NFкB, and hypoxia pathways by Phyllanthus prevents proliferation, metastasis and angiogenesis in human melanoma (MeWo) cancer cell line. Int J Med Sci 11 564-577, 2014.

58. Karin M: Nuclear factor-kappaB in cancer development and progression. Nature 441: 431-436, 2006.

59. Pacifico F and Leonardi A: Role of NF-kappaB in thyroid cancer. Mol Cell Endocrinol 321: 29-35, 2010

60. Beinke S and Ley SC: Functions of NF-kappaB1 and NF-kappaB2 in immune cell biology. Biochem J 382: 393-409, 2004.

61. Kucharczak J, Simmons MJ, Fan Y and Gélinas C: To be, or not to be: NF-kappaB is the answer--role of Rel/NF-kappaB in the regulation of apoptosis. Oncogene 22: 8961-8982, 2003.
62. Fu X, Fang J, Lian M, Zhong Q, Ma H, Feng L, Wang R and Wang H: Identification of microRNAs associated with medullary thyroid carcinoma by bioinformatics analyses. Mol Med Rep 15: 4266-4272, 2017

63. Chen Z, Yan B and Van Waes C: The role of the NF-kappaB transcriptome and proteome as biomarkers in human head and neck squamous cell carcinomas. Biomark Med 2: 409-426, 2008.

64. Durairajan S, Jebaraj Walter CE, Samuel MD, Palani D, G DJD, C GPD, Pasupati S and Johnson T: Differential expression of $\mathrm{NF}-\kappa \mathrm{B}$ heterodimer RelA/p50 in human urothelial carcinoma. PeerJ 6: e5563, 2018.

65. Qadir J, Riaz SK, Sahar NE, Aman D, Khan MJ and Malik MFA: Transcriptional elucidation of tumor necrosis factor- $\alpha$-mediated nuclear factor- $\kappa \mathrm{B} 1$ activation in breast cancer cohort of Pakistan. J Cancer Res Ther 16: 1443-1448, 2020.

66. Honma M, Kato N, Hashimoto M, Takahashi H, Ishida-Yamamoto A and Iizuka H: Subungual keratoacanthoma: Analysis of cell proliferation and copy number variation of oncogenes compared with periungual squamous cell carcinoma. Clin Exp Dermatol 36: 57-62, 2011.

67. Li S, Wang C, Yu X, Wu H, Hu J, Wang S and Ye Z: miR-3619-5p inhibits prostate cancer cell growth by activating CDKN1A expression. Oncol Rep 37: 241-248, 2017.

68. Zhang M, Luo H and Hui L: miR-3619-5p hampers proliferation and cisplatin resistance in cutaneous squamous-cell carcinoma via KPNA4. Biochem Biophys Res Commun 513: 419-425, 2019.

69. Hao P, Yue F, Xian X, Ren Q, Cui H and Wang Y: Inhibiting effect of MicroRNA-3619-5p/PSMD10 axis on liver cancer cell growth in vivo and in vitro. Life Sci 254: 117632, 2020.

This work is licensed under a Creative Commons Attribution-NonCommercial-NoDerivatives 4.0 International (CC BY-NC-ND 4.0) License. 